\title{
An Implementation of the QMR Method Based on Coupled Two-Term Recurrences
}

Roland W. Freund and Noël M. Nachtigal

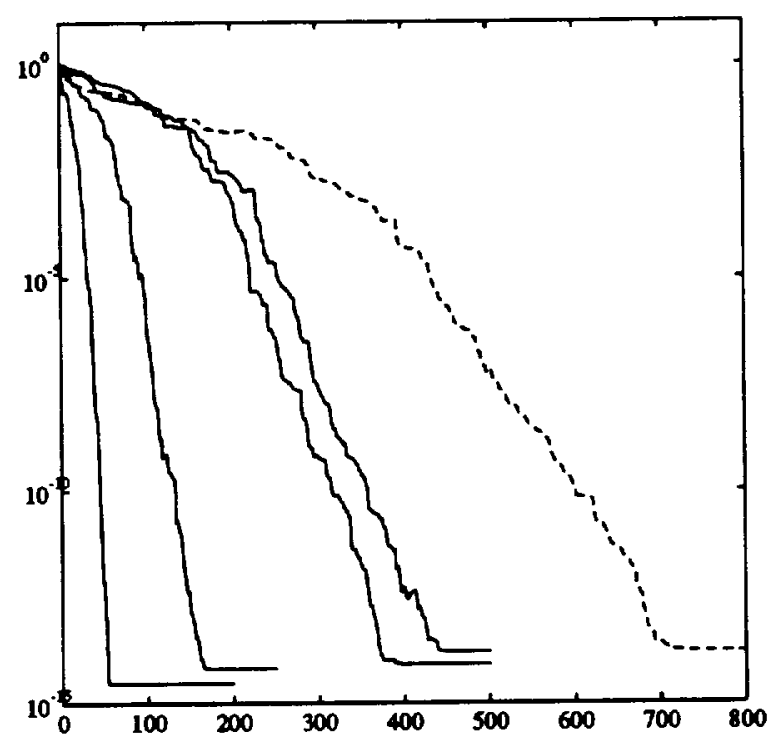

RIACS Technical Report 92.15

June 1992

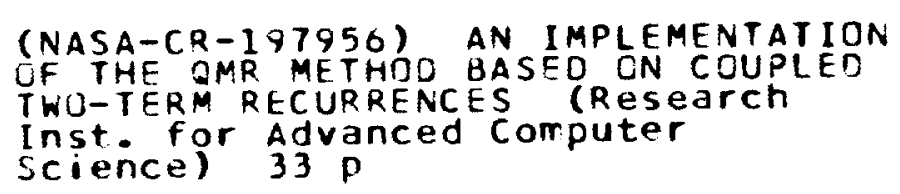





\title{
An Implementation of the QMR Method Based on Coupled Two-Term Recurrences
}

\author{
Roland W. Freund and Noël M. Nachtigal
}

The Research Institute for Advanced Computer Science is operated by Universities Space Research Association (USRA),

The American City Building, Suite 311, Columbia, MD 21044, (301)730-2656.

Work reported herein was supported in part by DARPA via Cooperative Agreement NCC 2-387 between NASA and USRA. 



\title{
AN IMPLEMENTATION OF THE QMR METHOD BASED ON COUPLED TWO-TERM RECURRENCES*
}

\author{
ROLAND W. FREUND ${ }^{\dagger}$ AND NOËL M. NACHTIGAL $\ddagger$
}

\begin{abstract}
Recently, the authors have proposed a new Krylov subspace iteration, the quasi-minimal residual algorithm (QMR), for solving non-Hermitian linear systems. In the original implementation of the QMR method, the Lanczos process with look-ahead is used to generate basis vectors for the underlying Krylov subspaces. In the Lanczos algorithm, these basis vectors are computed by means of three-term recurrences. It has been observed that, in finite precision arithmetic, vector iterations based on three-term recursions are usually less robust than mathematically equivalent coupled two-term vector recurrences.

This paper presents a look-ahead algorithm that constructs the Lanczos basis vectors by means of coupled two-term recursions. Implementation details are given, and the look-ahead strategy is described. A new implementation of the QMR method, based on this coupled two-term algorithm, is proposed. A simplified version of the QMR algorithm without look-ahead is also presented, and the special case of QMR for complex symmetric linear systems is considered. Results of numerical experiments comparing the original and the new implementations of the QMR method are reported.
\end{abstract}

Key words. Krylov subspace iteration, quasi-minimal residual method, non-Hermitian matrices, coupled two-term recurrences, look-ahead techniques, complex symmetric matrices

AMS(MOS) subject classifications. $65 \mathrm{~F} 10,65 \mathrm{~N} 22$

1. Introduction. Recently, we have proposed a new Krylov subspace iteration, the quasi-minimal residual algorithm (QMR) [9], for solving general nonsingular nonHermitian systems of linear equations

$$
A x=b .
$$

The QMR method is closely related to the classical biconjugate gradient algorithm (BCG) due to Lanczos [14]. The BCG method aims at generating approximate solutions for (1.1) that satisfy a Galerkin condition. Unfortunately, for non-Hermitian matrices $A$, such iterates need not always exist, and this is the source of one of the two possible breakdowns-triggered by division by 0 -that can occur during each iteration step of BCG. The second breakdown is equivalent to the possible breakdown-also triggered by division by 0 - of the nonsymmetric Lanczos process [13]. In finite precision arithmetic, it is unlikely that one encounters exact breakdowns in the BCG algorithm. However, near-breakdowns can occur, which can cause a build-up of round-off in successive iterations. Another problem with BCG is the lack of a residual minimization

- This work was supported by Cooperative Agreement NCC 2-387 between the National Aeronautics and Space Administration (NASA) and the Universities Space Research Association (USRA).

$\dagger$ AT\&T Bell Laboratories, Room 2C-420, 600 Mountain Avenue, P.O. Box 636, Murray Hill, New Jersey 07974-0636. This research was performed while this author was in residence at the Research Institute for Advanced Computer Science (RIACS), NASA Ames Research Center, Moffett Field, California 94035.

t RIACS, Mail Stop T041-5, NASA Ames Research Center, Moffett Field, California 94035. 
property for its iterates, which leads to a typically erratic convergence behavior, with wild oscillations in the residual norm.

The QMR method offers remedies for these problems. It generates iterates that are defined by a quasi-minimization of the residual norm, rather than a Galerkin condition. This eliminates the oscillations and leads to a smooth and nearly monotone convergence behavior. In contrast to BCG, a QMR iterate always exists at each iteration step, and this excludes breakdowns caused by non-existent iterates. Moreover, possible breakdowns in the underlying Lanczos process are prevented by using look-ahead techniques. Therefore, except for the rare event of an incurable breakdown, breakdowns cannot occur in the QMR method.

In the original QMR algorithm [9], an implementation of the Lanczos method with look-ahead is used to generate basis vectors for the underlying Krylov subspaces. In the Lanczos process, these basis vectors are generated by means of three-term recurrences. It has been observed that, in finite precision arithmetic, vector iterations based on three-term recursions are usually less robust than mathematically equivalent coupled two-term vector recurrences. In this paper, we present a general look-ahead algorithm based on coupled two-term recursions for constructing basis vectors of Krylov subspaces. Based on this algorithm we then propose a new implementation of the QMR method.

The remainder of the paper is organized as follows. In $\S 2$, we briefly review the Lanczos process and the original QMR algorithm. In $\S 3$, we present a sketch of the proposed look-ahead procedure for constructing Lanczos vectors by means of coupled two-term recurrences. In $\S 4$, we discuss the look-ahead strategy for this algorithm, and in $\S 5$, we give implementation details. Next we combine the coupled two-term procedure with the QMR approach. In $\S 6$, we outline the resulting implementation for the general case of QMR with look-ahead. In $\S 7$, we present a simplified version of the QMR algorithm without look-ahead. In $\S 8$, we consider a variant of $Q M R$ for the special case of complex symmetric linear systems. In $\S 9$, we report results of numerical experiments comparing the original and the new implementations of the QMR method. Finally, in $\S 10$, we make some concluding remarks.

Throughout the paper, all vectors and matrices are allowed to have real or complex entries. As usual, $M^{T}=\left[m_{k j}\right]$ and $M^{H}=\left[\bar{m}_{k j}\right]$ denote the transpose and the conjugate transpose, respectively, of the matrix $M=\left[m_{j k}\right]$. We use $\sigma_{\max }(M)$ and $\sigma_{\min }(M)$ for the largest and smallest singular value of $M$, respectively. The vector norm $\|x\|:=\sqrt{x^{H} x}$ is always the Euclidean norm and $\|M\|:=\sigma_{\max }(M)$ is the corresponding matrix norm. We denote by

$$
\mathcal{P}_{n}:=\left\{\varphi(\lambda) \equiv \gamma_{0}+\gamma_{1} \lambda+\cdots+\gamma_{n} \lambda^{n} \mid \gamma_{0}, \gamma_{1}, \cdots, \gamma_{n} \in C\right\}
$$

the set of all complex polynomials of degree at most $n$. The $n$th Krylov subspace of $C^{N}$ generated by $c \in C^{N}$ and the $N \times N$ matrix $B$ is defined by

$$
K_{n}(c, B):=\operatorname{span}\left\{c, B c, \cdots, B^{n-1} c\right\},
$$

and we will make use of the fact that

$$
K_{n}(c, B)=\left\{\varphi(B) c \mid \varphi \in \mathcal{P}_{n-1}\right\}
$$


Furthermore, it is always assumed that $A$ is an $N \times N$ matrix, singular or nonsingular.

Finally, we remark that, for complex matrices, there are two equivalent formulations of the Lanczos process, using either $A^{T}$ or $A^{H}$. In this paper, we have chosen the formulation with $A^{T}$, for two reasons. First, it avoids complex conjugation of the scalars in some of the recurrence relations, and second, the recursions reduce immediately for the special case of complex symmetric matrices.

2. The $Q M R$ algorithm. In this section, we briefly describe the $Q M R$ method and its original implementation [9]. We remark that, in [9], QMR was proposed for nonsingular linear systems (1.1). Freund and Hochbruck [8] showed that the QMR method can also be applied to singular square systems, and that it always generates welldefined iterates. However, as discussed in [8], these iterates converge to a meaningful solution of (1.1) only for consistent systems with coefficient matrices of index 1. An important special case for which these conditions are satisfied is consistent singular systems with a one-dimensional null space, i.e.,

$$
b \in\left\{A x \mid x \in \mathrm{C}^{N}\right\} \text { and } \operatorname{dim}\left\{x \in \mathrm{C}^{N} \mid A x=0\right\}=1 .
$$

In this paper, we always consider the QMR method for the general case of $N \times N$ linear systems, with singular or nonsingular coefficient matrices $A$.

2.1. Krylov subspace methods. Let $x_{0} \in \mathrm{C}^{N}$ be an arbitrary initial guess for the linear system (1.1), and denote by $r_{0}:=b-A x_{0}$ the corresponding residual vector. An iterative scheme for solving (1.1) is called a Krylov subspace method if, for any choice of $x_{0}$, it produces approximate solutions of the form

$$
x_{n} \in x_{0}+K_{n}\left(r_{0}, A\right), \quad n=1,2, \cdots .
$$

Clearly, the design of a Krylov subspace algorithm consists of two main parts: the construction of suitable basis vectors for the Krylov subspaces $K_{n}\left(r_{0}, A\right)$ in $(2.2)$, and the choice of the actual iterates $x_{n}$. The QMR method is an example of a Krylov subspace iteration, where the basis vectors are generated by means of the nonsymmetric Lanczos process, and the iterates are characterized by a quasi-minimal residual property. Next, we describe these two main ingredients of QMR.

2.2. The Lanczos process. The Lanczos method is started with two vectors,

$$
v_{1}=r_{0} / \rho_{1}, \text { where } \rho_{1}=\left\|r_{0}\right\|,
$$

and an arbitrary second starting vector

$$
w_{1} \in C^{N} \text { with }\left\|w_{1}\right\|=1 .
$$

It then produces two sequences of vectors

$$
\left\{v_{j}\right\}_{j=1}^{n} \text { and }\left\{w_{j}\right\}_{j=1}^{n}
$$


such that, for $n=1,2, \cdots$,

$$
\begin{aligned}
\operatorname{span}\left\{v_{1}, v_{2}, \cdots, v_{n}\right\} & =K_{n}\left(v_{1}, A\right), \\
\operatorname{span}\left\{w_{1}, w_{2}, \cdots, w_{n}\right\} & =K_{n}\left(w_{1}, A^{T}\right),
\end{aligned}
$$

and the two sets are biorthogonal or block biorthogonal, i.e.,

$$
W_{n}^{T} V_{n}=D_{n}
$$

where $D_{n}$ is a diagonal or block diagonal matrix. Here and in the sequel, we denote by

$$
V_{n}:=\left[\begin{array}{llll}
v_{1} & v_{2} & \cdots & v_{n}
\end{array}\right] \text { and } W_{n}:=\left[\begin{array}{llll}
w_{1} & w_{2} & \cdots & w_{n}
\end{array}\right]
$$

the matrices containing the Lanczos vectors $\left\{v_{j}\right\}_{j=1}^{n}$ and $\left\{w_{j}\right\}_{j=1}^{n}$ as columns. We remark that the conditions (2.6)-(2.7) determine the vectors (2.5) only up to scaling. Throughout this paper, we always scale the Lanczos vectors to have unit length:

$$
\left\|v_{n}\right\|=\left\|w_{n}\right\|=1, \quad n=1,2, \cdots
$$

The crucial point of the Lanczos process is that vectors satisfying (2.6)-(2.7) can be constructed by means of short vector recursions. In the classical Lanczos algorithm [13], the vectors are generated using simple three-term recurrences:

$$
\begin{aligned}
& \tilde{v}_{n+1}=A v_{n}-v_{n} \mu_{n}-v_{n-1} \nu_{n}, \\
& \quad \rho_{n+1}=\left\|\tilde{v}_{n+1}\right\|, \quad v_{n+1}=\tilde{v}_{n+1} / \rho_{n+1}, \\
& \tilde{w}_{n+1}=A^{T} w_{n}-w_{n} \mu_{n}-w_{n-1}\left(\nu_{n} \rho_{n} / \xi_{n}\right) \\
& \quad \xi_{n+1}=\left\|\tilde{w}_{n+1}\right\|, \quad w_{n+1}=\tilde{w}_{n+1} / \xi_{n+1}, \\
& \text { where } \quad \mu_{n}=w_{n}^{T} A v_{n} / w_{n}^{T} v_{n}, \quad \nu_{n}=\xi_{n} w_{n}^{T} v_{n} / w_{n-1}^{T} v_{n-1} .
\end{aligned}
$$

In this case, the matrix $D_{n}$ in (2.7) is diagonal and nonsingular:

$$
D_{n}=\operatorname{diag}\left(\delta_{1}, \delta_{2}, \cdots, \delta_{n}\right), \quad \text { where } \delta_{j}:=w_{j}^{T} v_{j} \neq 0 \text {. }
$$

Furthermore, we note that, using the notation introduced in (2.8), the recurrence relations $(2.10)-(2.11)$ for the first $n+1$ Lanczos vectors $\left\{v_{j}\right\}_{j=1}^{n+1}$ and $\left\{w_{j}\right\}_{j=1}^{n+1}$ can be written compactly in matrix form:

$$
\begin{aligned}
A V_{n} & =V_{n+1} H_{n}, \\
A^{T} W_{n} & =W_{n+1} \Gamma_{n+1}^{-1} H_{n} \Gamma_{n} .
\end{aligned}
$$

Here, $H_{n}$ is an $(n+1) \times n$ tridiagonal matrix, and

$$
\Gamma_{n}:=\operatorname{diag}\left(\gamma_{1}, \gamma_{2}, \cdots, \gamma_{n}\right), \quad \text { where } \quad \gamma_{j}:= \begin{cases}1 & \text { if } j=1 \\ \gamma_{j-1} \rho_{j} / \xi_{j} & \text { if } j>1\end{cases}
$$


is a diagonal scaling matrix with positive diagonal entries. Finally, for later use, we note that all subdiagonal elements of $H_{n}$ are nonzero, and therefore

$$
\operatorname{rank} H_{n}=n \text {. }
$$

Unfortunately, in the classical Lanczos algorithm, breakdowns cannot be excluded. Indeed, by (2.12), division by 0 will occur during the construction of $v_{n+1}$ and $w_{n+1}$ if $w_{n}^{T} v_{n}=0$, but $w_{n} \neq 0$ and $v_{n} \neq 0$. Parlett, Taylor, and Liu [15] were the first to devise a practical modification of the Lanczos procedure that uses look-ahead to skip over possible exact breakdowns $\left(w_{n}^{T} v_{n}=0\right)$ or near-breakdowns $\left(w_{n}^{T} v_{n}\right.$ is nonzero, but small in some sense). The QMR algorithm is based on a different implementation of the lookahead Lanczos method, recently developed by Freund, Gutknecht, and Nachtigal [7]. Next, we briefly sketch this look-ahead Lanczos procedure.

As in the classical Lanczos algorithm, two sequences of Lanczos vectors $\left\{v_{j}\right\}_{j=1}^{n}$ and $\left\{w_{j}\right\}_{j=1}^{n}$ are generated, starting with (2.3) and (2.4). Again, we will use the matrix notation $V_{n}$ and $W_{n}$ defined in (2.8). As before, these vectors satisfy (2.6)-(2.7), but now $D_{n}$ is, in general, only a block diagonal matrix, with $l:=l(n)$ square blocks of dimension $h_{j}, j=1,2, \cdots, l$, on the diagonal. More precisely, we have

$$
D_{n}=\operatorname{diag}\left(D^{(1)}, D^{(2)}, \cdots, D^{(l)}\right), \quad \text { where } \quad D^{(j)}:=\left(W^{(j)}\right)^{T} V^{(j)}
$$

Here, the matrices $V^{(j)}$ and $W^{(j)}, j=1,2, \cdots, l$, are defined by partitioning $V_{n}$ and $W_{n}$ into blocks, according to the look-ahead steps taken:

$$
V_{n}=\left[\begin{array}{llll}
V^{(1)} & V^{(2)} & \ldots & V^{(l)}
\end{array}\right] \text { and } \quad W_{n}=\left[\begin{array}{lllll}
W^{(1)} & W^{(2)} & \ldots & W^{(l)}
\end{array}\right] .
$$

We remark that the matrices $V^{(j)}$ and $W^{(j)}$ are of size $N \times h_{j}$, and they contain as their columns the Lanczos vectors constructed in the $j$ th look-ahead step. The integer $h_{j}$ is called the length of the $j$ th look-ahead step, and $l$ in (2.18)-(2.19) is the number of look-ahead steps that have been performed during the first $n$ steps of the Lanczos process. For later use, we introduce some further notation. For $j=1,2, \cdots$, we denote by $n_{j}$ the index of the first vectors of the blocks $V^{(j)}$ and $W^{(j)}$ in $(2.19)$; hence, we have

$$
V^{(j)}=\left[\begin{array}{llll}
v_{n,} & v_{n,+1} & \cdots
\end{array}\right] \text { and } W^{(j)}=\left[\begin{array}{lll}
w_{n_{j}} & w_{n_{j}+1} & \cdots
\end{array}\right] .
$$

Note that the indices $n_{j}$ satisfy

$$
1=: n_{1}<n_{2}<\cdots<n_{l} \leq n<n_{l+1}
$$

The vectors $v_{n_{j}}$ and $w_{n_{j}}$ are called regular, while the remaining vectors in the blocks $V^{(j)}$ and $W^{(j)}$ are called inner. We remark that, in view of (2.7) and (2.18), the regular vectors are biorthogonal to all previous Lanczos vectors, i.e.,

$$
w_{i}^{T} v_{n,}=w_{n j}^{T} v_{i}=0 \text { for all } i=1,2, \cdots, n_{j}-1,
$$

while the inner vectors in the $j$ th blocks $V^{(j)}$ and $W^{(j)}$ are biorthogonal to all Lanczos vectors from the previous blocks, but not necessarily to the Lanczos vectors in the $j$ th 
blocks. Finally, we note that, in $(2.18)$, the blocks $D^{(j)}, j=1,2, \cdots, l-1$, are all nonsingular, while the last block $D^{(l)}$ is nonsingular if $n_{l+1}=n+1$, i.e., if $v_{n+1}$ and $w_{n+1}$ are constructed as regular vectors.

In the look-ahead algorithm, the Lanczos vectors (2.5) are again generated using only short vector recurrences, which now involve vectors from the last two blocks $V^{(l)}, V^{(l-1)}$ and $W^{(l)}, W^{(l-1)}$, instead of just $v_{n}, v_{n-1}$ and $w_{n}, w_{n-1}$, as in the classical algorithm. For example, $v_{n+1}$ is computed by means of the relations

$$
\begin{aligned}
& \tilde{v}_{n+1}=A v_{n}-V^{(I)} \mu_{n}-V^{(l-1)} \nu_{n}, \\
& \rho_{n+1}=\left\|\tilde{v}_{n+1}\right\|, \quad v_{n+1}=\tilde{v}_{n+1} / \rho_{n+1},
\end{aligned}
$$

where $\mu_{n} \in C^{h_{l}}$ and $\nu_{n} \in C^{h_{l-1}}$ are suitable coefficient vectors. The second Lanczos vector $w_{n+1}$ is obtained similarly. As before, the recurrence relations for the Lanczos vectors can be summarized in the form (2.14)-(2.15). Here, $H_{n}$ is now a block tridiagonal matrix with $l$ square blocks on the diagonal, where the $j$ th block has dimension $h_{j} \times h_{j}, j=1,2, \cdots, l$. In addition, $H_{n}$ is also an upper Hessenberg matrix. Furthermore, (2.16) and (2.17) remain valid, and $\rho_{j}$ and $\xi_{j}$ in (2.16) are scaling factors used to ensure the normalization (2.9) of the Lanczos vectors, see (2.23).

We would like to stress that the look-ahead strategy (see [7] and also $\S 4$ below) is such that the algorithm performs mostly standard Lanczos steps, i.e., look-ahead steps of length $h_{j}=1$ with blocks $V^{(j)}$ and $W^{(j)}$ that consist of only single Lanczos vectors. True look-ahead steps, i.e., steps of size $h_{j}>1$, are only used to avoid exact and nearbreakdowns. Typically, except for contrived examples, only few true look-ahead steps occur, and their size is usually small, mostly $h_{j}=2$. We note that, if only steps of length $h_{j}=1$ are performed, then the look-ahead Lanczos algorithm reduces to the classical algorithm. Finally, we remark that so-called incurable breakdowns $[18,12]$ can occur in the Lanczos process. Such breakdowns cannot be remedied by look-ahead, and indeed, in such a case, the look-ahead Lanczos algorithm would build a block of size $h_{l}=\infty$. Fortunately, incurable breakdowns are extremely rare, and they do not present a problem in practice.

The look-ahead Lanczos algorithm is intimately connected with formally orthogonal polynomials, see, e.g., [12, 7]. In particular, we will use the fact that each pair of Lanczos vectors $v_{j}$ and $w_{j}$ can be expressed in the form

$$
v_{j}=\phi_{j-1}(A) v_{1} \text { and } w_{j}=\gamma_{j} \phi_{j-1}\left(A^{T}\right) w_{1}
$$

where $\phi_{j-1} \in \mathcal{P}_{j-1}$ is of exact degree $j-1$ and $\gamma_{j}>0$ is defined in (2.16).

For further details and properties of the look-ahead Lanczos algorithm, we refer the reader to [7].

2.3. The quasi-minimal residual property. In the QMR method, the vectors $\left\{v_{j}\right\}_{j=1}^{n}$ generated by the look-ahead Lanczos algorithm are used as a basis for the Krylov subspace $K_{n}\left(r_{0}, A\right)$ in (2.2). The $n$th QMR iterate $x_{n}$ is then defined by

$$
x_{n}=x_{0}+V_{n} z_{n},
$$


where $z_{n} \in \mathrm{C}^{n}$ is the unique solution of the least squares problem

$$
\left\|f_{n+1}-\Omega_{n+1} H_{n} z_{n}\right\|=\min _{z \in \mathcal{C}^{n}}\left\|f_{n+1}-\Omega_{n+1} H_{n} z\right\| .
$$

Here

$$
f_{n+1}:=\omega_{1} \rho_{1} \cdot\left[\begin{array}{llll}
1 & 0 & \cdots & 0
\end{array}\right]^{T} \in \mathbf{R}^{n+1},
$$

with $\rho_{1}$ given in $(2.3)$, and

$$
\Omega_{n+1}:=\operatorname{diag}\left(\omega_{1}, \omega_{2}, \cdots, \omega_{n+1}\right), \quad \omega_{j}>0, \quad j=1,2, \cdots, n+1,
$$

is an arbitrary diagonal weighting matrix. Note that, in view of (2.17) and (2.28), the $(n+1) \times n$ matrix $\Omega_{n+1} H_{n}$ has full rank $n$. This guarantees that there always exists a unique solution of (2.26). Furthermore, we remark that the standard choice for the weights in $(2.28)$ is

$$
\omega_{j}=1 \text { for all } j \text {. }
$$

However, there are instances (see [10]) where the use of different weights is crucial, and therefore, we formulate the QMR method in the general setting (2.28).

From (2.25), (2.14), (2.27), and (2.3), it follows that the residual vector $r_{n}:=b-A x_{n}$ corresponding to $x_{n}$ satisfies

$$
r_{n}=V_{n+1} \Omega_{n+1}^{-1}\left(f_{n+1}-\Omega_{n+1} H_{n} z_{n}\right)
$$

Hence, in view of (2.26), the $n$th QMR iterate $x_{n}$ is characterized by a minimization of the second factor in (2.30); this is just the quasi-minimal residual property. The relation (2.30) shows that the scaling $(2.29)$ is very natural, in the sense that all columns of $V_{n+1} \Omega_{n+1}^{-1}$ in the representation (2.30) of $r_{n}$ are treated equally. We remark that the QMR iterates $x_{n}$ can be easily updated from step to step. Due to the block tridiagonal structure of $H_{n}$, this update can be implemented with only short recurrences; see [9] for details. Finally, we note that the quasi-minimal residual property can be used to derive convergence results for the QMR method; we refer the reader to $[9,6]$.

3. A coupled two-term procedure with look-ahead. In this section, we consider a different approach to constructing the Lanczos vectors. The basic idea is to break up the three-term recurrences in the Lanczos process into coupled two-term recurrences, by using -in addition to the Lanczos vectors-a suitable second set of basis vectors for the underlying Krylov subspaces. In $\S 9$, we will illustrate that QMR based on this coupled two-term procedure has better numerical properties than the original implementation of QMR based on three-term recurrences.

3.1. The general setting. In the following, let $\left\{v_{j}\right\}_{j=1}^{n}$ and $\left\{w_{j}\right\}_{j=1}^{n}$ always denote the sequence of vectors generated by the look-ahead Lanczos algorithm, as described in $\S 2.2$. We assume that we are also given a second set of basis vectors

$$
\left\{p_{j}\right\}_{j=1}^{n} \text { and }\left\{q_{j}\right\}_{j=1}^{n}
$$


for the Krylov subspaces $K_{n}\left(v_{1}, A\right)$ and $K_{n}\left(w_{1}, A^{T}\right)$. More precisely, we consider vectors (3.1) that-in analogy to (2.24)-are of the form

$$
p_{j}=\psi_{j-1}(A) v_{1} \quad \text { and } \quad q_{j}=\gamma_{j} \psi_{j-1}\left(A^{T}\right) w_{1},
$$

where $\gamma_{j}>0$ is given by $(2.16)$, and $\psi_{j-1} \in \mathcal{P}_{j-1}$ is of exact degree $j-1$ with the same leading coefficient as the polynomial $\phi_{j-1}$ in (2.24). To distinguish between the two bases, we will often refer to the Lanczos vectors $\left\{v_{j}\right\}_{j=1}^{n}$ and $\left\{w_{j}\right\}_{j=1}^{n}$ as the $V-W$ sequence and to the vectors (3.1) as the $P-Q$ sequence.

From (2.24) and (3.2), we conclude that, for each $n=1,2, \cdots$,

$$
\begin{aligned}
& p_{n}=v_{n}-\sum_{i=1}^{n-1} p_{i} u_{i n}, \\
& q_{n}=w_{n}-\sum_{i=1}^{n-1} q_{i} u_{i n}\left(\gamma_{n} / \gamma_{i}\right),
\end{aligned}
$$

with suitable coefficients $u_{\text {in }} \in \mathrm{C}, i=1,2, \ldots, n-1$. Similarly, in view of (2.23), (2.24), (3.2), and (2.16), we have

$$
\begin{aligned}
& \tilde{v}_{n+1}=A p_{n}-\sum_{i=1}^{n} v_{i} l_{i n}, \quad \rho_{n+1}=\left\|\tilde{v}_{n+1}\right\|, \quad v_{n+1}=\tilde{v}_{n+1} / \rho_{n+1}, \\
& \tilde{w}_{n+1}=A^{T} q_{n}-\sum_{i=1}^{n} w_{i} l_{i n}\left(\gamma_{n} / \gamma_{i}\right), \quad \xi_{n+1}=\left\|\tilde{w}_{n+1}\right\|, \quad w_{n+1}=\tilde{w}_{n+1} / \xi_{n+1},
\end{aligned}
$$

with suitable coefficients $l_{\text {in }} \in C, i=1,2, \ldots, n$. Note that (3.3)-(3.6) are coupled recurrences for generating the $P-Q$ and $V-W$ sequences: first, $p_{n}$ and $q_{n}$ are computed by means of (3.3)-(3.4), and then, the next Lanczos pair $v_{n+1}$ and $w_{n+1}$ is obtained from (3.5)-(3.6). Of course, it remains to specify the actual choice of the $P-Q$ sequence. In order to minimize work and storage of previous vectors, the goal here is to select these vectors such that the recurrences (3.3)-(3.6) are as short as possible.

It will be convenient to use-in addition to (2.8)-the notation

$$
P_{n}:=\left[\begin{array}{llll}
p_{1} & p_{2} & \cdots & p_{n}
\end{array}\right] \text { and } Q_{n}:=\left[\begin{array}{llll}
q_{1} & q_{2} & \cdots & q_{n}
\end{array}\right] \text {. }
$$

The recurrences (3.3)-(3.6) for the vectors $\left\{p_{j}\right\}_{j=1}^{n},\left\{q_{j}\right\}_{j=1}^{n},\left\{v_{j}\right\}_{j=1}^{n+1}$, and $\left\{w_{j}\right\}_{j=1}^{n+1}$ can then be written compactly in matrix form:

$$
\begin{array}{rlrl}
V_{n} & =P_{n} U_{n}, & A P_{n} & =V_{n+1} L_{n}, \\
W_{n} & =Q_{n} \Gamma_{n}^{-1} U_{n} \Gamma_{n}, & A^{T} Q_{n}=W_{n+1} \Gamma_{n+1}^{-1} L_{n} \Gamma_{n} .
\end{array}
$$

Here, $U_{n}$ is an upper triangular matrix and $L_{n}$ is an upper Hessenberg matrix given by

$$
U_{n}:=\left[\begin{array}{cccc}
1 & u_{12} & \cdots & u_{1 n} \\
0 & 1 & \ddots & \vdots \\
\vdots & \ddots & \ddots & u_{n-1, n} \\
0 & \cdots & 0 & 1
\end{array}\right] \text { and } L_{n}:=\left[\begin{array}{cccc}
l_{11} & l_{12} & \cdots & l_{1 n} \\
\rho_{2} & l_{22} & & \vdots \\
0 & \rho_{3} & \ddots & \vdots \\
\vdots & \ddots & \ddots & l_{n n} \\
0 & \cdots & 0 & \rho_{n+1}
\end{array}\right]
$$


respectively, and $\Gamma_{n}$ is the diagonal matrix defined in (2.16). Note that, by eliminating $P_{n}$ in (3.7), we obtain

$$
A V_{n}=V_{n+1} L_{n} U_{n}
$$

By comparing (3.10) with (2.14), it follows that

$$
H_{n}=L_{n} U_{n},
$$

i.e., the matrices (3.9) define a factorization of the block tridiagonal Hessenberg matrix $H_{n}$ generated by the look-ahead Lanczos algorithm.

Recall from (2.7) and (2.18) that the Lanczos vectors are block biorthogonal. These biorthogonality relations determine the coefficients $l_{\text {in }}$ in (3.5)-(3.6). For example, consider the case that $v_{n+1}$ and $w_{n+1}$ are constructed as regular vectors. Then, in view of (2.22), we have the condition $W_{n}^{T} v_{n+1}=0$, which, by (3.5), is equivalent to:

$$
0=W_{n}^{T} A p_{n}-\sum_{i=1}^{n} W_{n}^{T} v_{i} l_{i n} .
$$

Using (2.7), (2.8), and the first equation in (3.8), we deduce from (3.12) that

$$
\left[\begin{array}{c}
l_{1 n} \\
\vdots \\
l_{n n}
\end{array}\right]=D_{n}^{-1} \Gamma_{n} U_{n}^{T} \Gamma_{n}^{-1} Q_{n}^{T} A p_{n} .
$$

Recall from (2.18), (2.16), and (3.9) that the matrices $D_{n}^{-1}, \Gamma_{n}$, and $U_{n}^{T}$ are block diagonal, diagonal, and lower triangular, respectively. Hence the relation (3.13) implies that the vector $Q_{n}^{T} A p_{n}$ determines the length of the recurrences (3.5)-(3.6). In particular, in order to obtain recursions that are as short as possible, the $\mathrm{P}-\mathrm{Q}$ sequence should be chosen such that the vector $Q_{n}^{T} A p_{n}$ has as many leading zeros as possible. The same conclusion also holds for the case that $v_{n+1}$ and $w_{n+1}$ are constructed as inner vectors.

Motivated by this discussion, we require that the vectors in the $P-Q$ sequence be $A$-biorthogonal or block $A$-biorthogonal, in the sense that the matrix

$$
E_{n}:=Q_{n}^{T} A P_{n}
$$

should be diagonal or block diagonal. Note that the vector $Q_{n}^{T} A p_{n}$ is just the nth column of $E_{n}$. Furthermore, we remark that $q_{n}^{T} A P_{n}$, the $n$th row of $E_{n}$, has the same zero structure as $Q_{n}^{T} A p_{n}$. This is a consequence of relation (3.16) in the following lemma, which we will also need later on.

LEMMA 3.1. Let $\left\{v_{i}\right\}_{i=1}^{n},\left\{w_{i}\right\}_{i=1}^{n}$ and $\left\{p_{i}\right\}_{i=1}^{n},\left\{q_{i}\right\}_{i=1}^{n}$ be vectors satisfying (2.24) and (3.2), respectively, and let $\Gamma_{n}=\operatorname{diag}\left(\gamma_{1}, \gamma_{2}, \cdots, \gamma_{n}\right)$. Let $D_{n}$ and $E_{n}$ be the matrices given by (2.7) and (3.14), respectively. Then:

$$
\begin{aligned}
& D_{n} \Gamma_{n}=\left(D_{n} \Gamma_{n}\right)^{T} \\
& E_{n} \Gamma_{n}=\left(E_{n} \Gamma_{n}\right)^{T}
\end{aligned}
$$


Proof. Using the polynomial representation (2.24) for the V-W vectors and the fact that polynomials in a matrix commute, we obtain

$$
w_{i}^{T} v_{j} \gamma_{j}=\gamma_{i} w_{1}^{T} \phi_{i-1}(A) \phi_{j-1}(A) v_{1} \gamma_{j}=\gamma_{j} w_{1}^{T} \phi_{j-1}(A) \phi_{i-1}(A) v_{1} \gamma_{i}=w_{j}^{T} v_{i} \gamma_{i},
$$

and thus (3.15). The relation (3.16) follows similarly.

3.2. The coupled algorithm without look-ahead. Next, we briefly consider the case that the $\mathrm{V}-\mathrm{W}$ sequence consists of the vectors generated by the classical Lanczos algorithm without look-ahead. Recall from (2.13) that here the matrix $D_{n}$ in (2.7) is diagonal, and that the Lanczos vectors are biorthogonal:

$$
\boldsymbol{w}_{j}^{T} v_{n}= \begin{cases}0 & \text { if } j \neq n \\ \delta_{n} \neq 0 & \text { if } j=n\end{cases}
$$

Suppose that it is possible to construct the vectors in the $P-Q$ sequence such that the matrix $E_{n}$ in (3.14) is also diagonal:

$$
E_{n}=\operatorname{diag}\left(\epsilon_{1}, \epsilon_{2}, \cdots, \epsilon_{n}\right), \text { where } \quad \epsilon_{j}:=q_{j}^{T} A p_{j} \neq 0 .
$$

By (3.14) and (3.18), the $\mathrm{P}-\mathrm{Q}$ vectors are then $A$-biorthogonal:

$$
q_{j}^{T} A p_{n}= \begin{cases}0 & \text { if } j \neq n \\ \epsilon_{n} \neq 0 & \text { if } j=n\end{cases}
$$

With (2.13), (3.9), and (3.19), we deduce from (3.13) that

$$
l_{\text {in }}=0, \quad i=1,2, \cdots, n-1, \quad \text { and } \quad l_{n n}=\beta_{n}:=\epsilon_{n} / \delta_{n} .
$$

Furthermore, by multiplying (3.3) from the left by $q_{j}^{T} A$ and by using (3.19), (3.6), and (3.17), we obtain that, for $j=1,2, \cdots, n-1$,

$$
\begin{aligned}
0=q_{j}^{T} A p_{n} & =q_{j}^{T} A v_{n}-\epsilon_{j} u_{j n} \\
& =\left(A^{T} q_{j}\right)^{T} v_{n}-\epsilon_{j} u_{j n} \\
& =\xi_{j+1} w_{j+1}^{T} v_{n}-\epsilon_{j} u_{j n} .
\end{aligned}
$$

With (3.17), it follows from (3.21) that

$$
u_{i n}=0, \quad i=1,2, \cdots, n-2, \quad \text { and } u_{n-1, n}=\xi_{n} \delta_{n} / \epsilon_{n-1} .
$$

In view of (3.20) and (3.22), all but the last terms vanish in each of the sums in (3.3)(3.6), and hence (3.3)-(3.6) reduces to a coupled two-term procedure for generating the $P-Q$ and $V-W$ sequences.

The nth iteration of the the resulting algorithm can be summarized as follows. 
AlgorithM 3.2. ( $n$th iteration of the coupled algorithm without look-ahead)

1) If $\epsilon_{n-1}=0$, then stop.

Otherwise, compute $\delta_{n}=w_{n}^{T} v_{n}$.

If $\delta_{n}=0$, then stop.

2) Compute

$$
\begin{aligned}
& p_{n}=v_{n}-p_{n-1}\left(\xi_{n} \delta_{n} / \epsilon_{n-1}\right), \\
& q_{n}=w_{n}-q_{n-1}\left(\rho_{n} \delta_{n} / \epsilon_{n-1}\right) .
\end{aligned}
$$

3) Compute $\epsilon_{n}=q_{n}^{T} A p_{n}, \beta_{n}=\epsilon_{n} / \delta_{n}$, and set

$$
\begin{gathered}
\tilde{v}_{n+1}=A p_{n}-v_{n} \beta_{n}, \quad \rho_{n+1}=\left\|\tilde{v}_{n+1}\right\|, \\
\tilde{w}_{n+1}=A^{T} q_{n}-w_{n} \beta_{n}, \quad \xi_{n+1}=\left\|\tilde{w}_{n+1}\right\| .
\end{gathered}
$$

4) If $\rho_{n+1}=0$ or $\xi_{n+1}=0$, then stop.

Otherwise, set

$$
v_{n+1}=\tilde{v}_{n+1} / \rho_{n+1}, \quad w_{n+1}=\tilde{w}_{n+1} / \xi_{n+1}
$$

We remark that the vectors in the $P-Q$ and $V-W$ sequences are, up to scaling, just the search directions and the residual vectors generated by the BCG method [14, 4]. In particular, Algorithm 3.2 can be viewed as the $n$th iteration of a rescaled version of $\mathrm{BCG}$, where the computation of the $\mathrm{BCG}$ iterates is omitted.

In finite arithmetic, one of the termination checks in steps 1) or 4) of the coupled two-term procedure will be satisfied after at most $N$ iterations. Normally, the algorithm stops due to $\rho_{n+1}=0$ or $\xi_{n+1}=0$, and then the procedure has constructed a basis for the invariant subspaces $K_{n}\left(v_{1}, A\right)$ or $K_{n}\left(w_{1}, A^{T}\right)$, respectively. This is called regular termination. If $\epsilon_{n-1}=0$ or $\delta_{n}=0$ occurs, then the algorithm has to be stopped to avoid division by 0 . This is referred to as an exact breakdown. Recall from $\S 2.2$ that $\delta_{n}=0$ signals a breakdown in the classical Lanczos algorithm. Note that, like BCG, the coupled two-term procedure now has a second source of breakdown, namely the case $\epsilon_{n-1}=0$. It can be shown that the condition $\epsilon_{n-1}=0$ corresponds to a breakdown in the BCG algorithm due to an $n$th iterate not being defined by the Galerkin condition.

In finite precision arithmetic, exact breakdowns are rather unlikely. However, near breakdowns, where $\delta_{n}$ or $\epsilon_{n-1}$ is nonzero, but small in some sense, may occur, leading to numerical instabilities in subsequent iterations. Next, we sketch a coupled two-term procedure that uses look-ahead in the construction of both the V-W and $P-Q$ sequences to avoid exact and near-breakdowns.

3.3. The general algorithm with look-ahead. For describing the look-ahead in the $\mathrm{V}-\mathrm{W}$ sequence, we will use the notations $(2.18)-(2.21)$ introduced in $\S 2.2$. In particular, the integer $l:=l(n)$ denotes the number of look-ahead steps that have been performed during the construction of the first $n$ vectors $\left\{v_{i}\right\}_{i=1}^{n}$ and $\left\{w_{i}\right\}_{i=1}^{n}$ in the 
$\mathrm{V}-\mathrm{W}$ sequence, and the $n_{j}$ 's in (2.21) are the indices of the regular vectors. Recall that, by (2.7) and (2.18), the $V-W$ vectors satisfy the block biorthogonality conditions

$$
\left(W^{(i)}\right)^{T} V^{(j)}=\left\{\begin{array}{ll}
0 & \text { if } \quad i \neq j, \\
D^{(j)} & \text { if } \quad i=j,
\end{array} \quad i, j=1,2, \cdots, l .\right.
$$

Here, the blocks $D^{(j)}$ are all nonsingular, except for possibly $D^{(l)}$. However, we have that necessarily

$$
D^{(l)} \text { is nonsingular, if } n_{l+1}=n+1 \text {. }
$$

Next, we introduce similar notations for describing the look-ahead in the $\mathrm{P}-\mathrm{Q}$ sequence. We denote by $k:=k(n)$ the number of look-ahead steps that have been performed during the construction of the first $n-1$ vectors $\left\{p_{i}\right\}_{i=1}^{n-1}$ and $\left\{q_{i}\right\}_{i=1}^{n-1}$ in the $\mathrm{P}-\mathrm{Q}$ sequence. In analogy to (2.19), we partition these vectors into blocks, according to the look-ahead steps taken:

$$
P_{n-1}=\left[\begin{array}{llll}
P^{(1)} & P^{(2)} & \ldots & P^{(k)}
\end{array}\right] \quad \text { and } \quad Q_{n-1}=\left[\begin{array}{llll}
Q^{(1)} & Q^{(2)} & \cdots & Q^{(k)}
\end{array}\right] .
$$

Recall from (3.14) that the $\mathrm{P}-\mathrm{Q}$ vectors are constructed to be block $A$-biorthogonal. More precisely, we have

$$
E_{n-1}=\operatorname{diag}\left(E^{(1)}, E^{(2)}, \cdots, E^{(k)}\right)
$$

or, equivalently,

$$
\left(Q^{(i)}\right)^{T} A P^{(j)}=\left\{\begin{array}{ll}
0 & \text { if } \quad i \neq j, \\
E^{(j)} & \text { if } \quad i=j,
\end{array} \quad i, j=1,2, \cdots, k .\right.
$$

Here, the blocks $E^{(j)}$ are nonsingular, except for possibly the last block $E^{(k)}$. In analogy to $(2.21)$, we denote by $m_{j}$ the indices of the first vectors of the blocks $P^{(j)}$ and $Q^{(j)}$ in (3.25). Hence, for $j=1,2, \cdots, k$, we have

$$
P^{(j)}=\left[\begin{array}{lll}
p_{m,} & p_{m,+1} & \cdots
\end{array}\right] \text { and } Q^{(j)}=\left[\begin{array}{lll}
q_{m,} & q_{m,+1} & \cdots
\end{array}\right] .
$$

Furthermore, the indices $m_{j}$ satisfy

$$
1=: m_{1}<m_{2}<\cdots<m_{k}<n \leq m_{k+1} \text {. }
$$

We remark that, by (3.26) and (3.27), the vectors $p_{m,}$ and $q_{m,}$ are $A$-biorthogonal to all previous $P-Q$ vectors, i.e.,

$$
q_{i}^{T} A p_{m_{j}}=q_{m}^{T} A p_{i}=0 \text { for all } i=1,2, \cdots, m_{j}-1 \text {. }
$$

Therefore, using the same notation as for the V-W sequence, we refer to the vectors $p_{m}$ and $q_{m}$ as regular vectors, while the remaining vectors in (3.27) are called inner. Finally, it turns out that, in (3.26), the last block $E^{(k)}$ has to be nonsingular, if $p_{n}$ and $q_{n}$ are constructed as regular vectors. This means that, in analogy to (3.24),

$$
E^{(k)} \text { is nonsingular, if } m_{k+1}=n \text {. }
$$


After these preliminaries, we can now sketch the actual algorithm. Let $n \geq 1$, and assume that we have already generated the first $n-1$ vectors $\left\{p_{i}\right\}_{i=1}^{n-1}$ and $\left\{q_{i}\right\}_{i=1}^{n-1}$ of the $\mathrm{P}-\mathrm{Q}$ sequence, and the first $n$ vectors $\left\{v_{i}\right\}_{i=1}^{n}$ and $\left\{w_{i}\right\}_{i=1}^{n}$ of the $\mathrm{V}-\mathrm{W}$ sequence.

First, the next pair of $\mathrm{P}-\mathrm{Q}$ vectors, $p_{n}$ and $q_{n}$, is constructed, using the recurrences (3.3)-(3.4). Here, the coefficients $u_{\text {in }}$ need to be chosen such that $p_{n}$ satisfies the corresponding $A$-biorthogonality conditions (3.26). We note that, in view of (3.16), the $A$-biorthogonality relations for the vector $q_{n}$ are then also fulfilled. With (3.6) and (3.23), one readily verifies that

$$
q_{i}^{T} A v_{n}=\left(A^{T} q_{i}\right)^{T} v_{n}=0 \text { for all } i=1,2, \cdots, n_{l}-2 .
$$

By rewriting this in terms of the blocks $Q^{(j)}$, we obtain that

$$
\left(Q^{(j)}\right)^{T} A v_{n}=0 \text { for all } j=1,2, \cdots, k^{\star}-1,
$$

where $k^{\star}:=k^{\star}(n)$ is given by

$$
k^{\star}=\max \left\{j \mid 1 \leq j \leq k \text { and } m_{j} \leq \max \left\{1, n_{l}-1\right\}\right\} .
$$

The orthogonality conditions (3.30), together with (3.26), imply that, in (3.3), we have $u_{\text {in }}=0$ for all $i<m_{k^{*}}$. Thus the recurrences (3.3)-(3.4) reduce to

$$
\begin{aligned}
& p_{n}=v_{n}-\sum_{i=m_{k^{*}}}^{n-1} p_{i} u_{i n}, \\
& q_{n}=w_{n}-\sum_{i=m_{k^{\star}}}^{n-1} q_{i} u_{i n}\left(\gamma_{n} / \gamma_{i}\right) .
\end{aligned}
$$

Now we need to determine the coefficients $u_{\text {in }}$ for $m_{k^{*}} \leq i \leq n-1$. This is done by enforcing the remaining $A$-biorthogonality conditions (3.26) for $p_{n}$ and the vectors $q_{i}$, namely

$$
q_{i}^{T} A p_{n}=0 \text { for all } i=m_{k^{\star}}, m_{k^{\star}}+1, \cdots, m^{\star} .
$$

Here, $m^{\star}:=n-1$ if $p_{n}$ and $q_{n}$ are constructed as regular vectors, and $m^{\star}:=m_{k}-1$ otherwise. Note that, in view of (3.28), we always have $m_{k}-1 \leq m^{\star}$. First, we consider (3.34) for the indices $i$ in the range $m_{k^{\star}} \leq i \leq m_{k}-1$. Using (3.32) and (3.26), we deduce from (3.34) that

$$
U_{m_{k} * m_{k}-1, n}=\left(\begin{array}{lllll}
\operatorname{diag}\left(E^{\left(k^{*}\right)}\right. & \cdots & \left.E^{(k-1)}\right)
\end{array}\right)^{-1}\left[\begin{array}{lll}
Q^{\left(k^{*}\right)} & \cdots & Q^{(k-1)}
\end{array}\right]^{T} A v_{n} .
$$

Here and in the sequel, we use the notation

$$
M_{i: j, n}:=\left[\begin{array}{llll}
m_{i n} & m_{i+1, n} & \cdots & m_{j n}
\end{array}\right]^{T}
$$

for vectors consisting of successive elements of the $n$th column of the matrix $M=\left[m_{i j}\right]$, and similarly, we denote by $M_{n, i: j}$ row vectors consisting of successive entries of the $n$th 
row of $M$. If $p_{n}$ and $q_{n}$ are constructed as regular vectors, then we also have to ensure that (3.34) holds for $i$ with $m_{k} \leq i \leq n-1$, and this gives

$$
U_{m_{k}: n-1, n}=\left(E^{(k)}\right)^{-1}\left(Q^{(k)}\right)^{T} A v_{n} .
$$

We remark that, by (3.29), the matrix $E^{(k)}$ in (3.36) is necessarily nonsingular since the vectors $p_{n}$ and $q_{n}$ are regular. If $p_{n}$ and $q_{n}$ are constructed as inner vectors, then $m^{\star}=m_{k}-1$, and (3.34) yields no conditions for the choice of the coefficients $u_{\text {in }}$ with $m_{k} \leq i<n-1$. In this case, we set

$$
u_{\text {in }}=\zeta_{\text {in }} \text { for } \quad i=m_{k}, m_{k}+1, \cdots, n-1,
$$

where $\zeta_{\text {in }} \in \mathrm{C}$ can be chosen arbitrarily. Finally, if $p_{n}$ and $q_{n}$ are regular, we update the "regular" indices (3.28) by setting $m_{k+1}:=n$ and $k:=k+1$. This completes the construction of the $p_{n}$ and $q_{n}$ vectors.

In a second step, we now compute the next pair of $V-W$ vectors, $v_{n+1}$ and $w_{n+1}$, using the recurrences (3.5)-(3.6) Here, we have to determine the coefficients $l_{\text {in }}$ such that $v_{n+1}$ satisfies the corresponding biorthogonality conditions (3.23). Note that, in view of (3.15), the relations (3.23) for $w_{n+1}$ are then fulfilled automatically. We proceed similar to the construction of $p_{n}$ and $q_{n}$. By using (3.26) and the fact that the columns of the matrices $Q_{i}$ and $W_{i}$ span the same space, it is easily verified that

$$
\left(W^{(j)}\right)^{T} A p_{n}=0 \text { for all } j=1,2, \cdots, l^{\star}-1,
$$

where $l^{\star}:=l^{\star}(n)$ is given by

$$
l^{\star}=\max \left\{j \mid 1 \leq j \leq l \text { and } n_{j} \leq m_{k}\right\} .
$$

From (3.38), we conclude that the recurrences (3.5)-(3.6) reduce as follows:

$$
\begin{aligned}
& \tilde{v}_{n+1}=A p_{n}-\sum_{i=n_{l^{\star}}}^{n} v_{i} l_{i n}, \\
& \tilde{w}_{n+1}=A^{T} q_{n}-\sum_{i=n_{l \star}}^{n} w_{i} l_{i n}\left(\gamma_{n} / \gamma_{i}\right) .
\end{aligned}
$$

The recurrence coefficients $l_{\text {in }}$ in (3.40)-(3.41) are determined by enforcing the remaining biorthogonality conditions (3.23) for the vectors $v_{n+1}$ and $w_{i}, n_{l^{*}} \leq i \leq n$. This gives

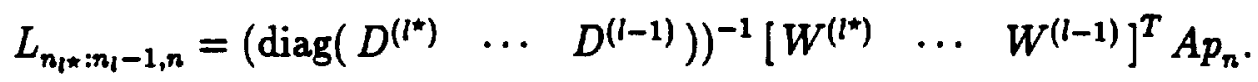

Moreover, if $v_{n+1}$ and $w_{n+1}$ are constructed as regular vectors, then

$$
L_{n ! n, n}=\left(D^{(l)}\right)^{-1}\left(W^{(l)}\right)^{T} A p_{n} .
$$


Note that, by (3.24), the matrix $D^{(l)}$ in (3.43) is necessarily nonsingular since $v_{n+1}$ and $w_{n+1}$ are regular. If $v_{n+1}$ and $w_{n+1}$ are built as inner vectors, then we set

$$
l_{\text {in }}=\eta_{\text {in }} \text { for } i=n_{l}, n_{l}+1, \cdots, n,
$$

where $\eta_{i n} \in C$ can be chosen arbitrarily. Finally, if $v_{n+1}$ and $w_{n+1}$ are regular, then we update the indices $(2.21)$ by setting $n_{l+1}:=n+1$ and $l:=l+1$.

The resulting coupled procedure for generating the $P-Q$ and $V-W$ sequences can be sketched as follows.

Algorithm 3.3. (Coupled algorithm with look-ahead)

0) Choose $v_{1}, w_{1} \in \mathrm{C}^{N}$ with $\left\|v_{1}\right\|=\left\|w_{1}\right\|=1$.

Set $V^{(1)}=v_{1}, W^{(1)}=w_{1}, D^{(1)}=w_{1}^{T} v_{1}$.

Set $k=1, m_{k}=1, l=1, n_{l}=1$.

For $n=1,2, \cdots$, do:

1) Determine $k^{\star}$ from (3.31).

2) Decide whether to construct $p_{n}$ and $q_{n}$ as regular or inner vectors and go to 3) or 4), respectively.

3) Compute $p_{n}$ and $q_{n}$ by means of (3.35)-(3.36) and (3.32)-(3.33).

Set $m_{k+1}=n, k=k+1, P^{(k)}=Q^{(k)}=\emptyset$ and go to 5$)$.

4) Compute $p_{n}$ and $q_{n}$ by means of (3.35), (3.37), and (3.32)-(3.33).

5) Set

$$
P^{(k)}=\left[\begin{array}{ll}
P^{(k)} & p_{n}
\end{array}\right], \quad Q^{(k)}=\left[\begin{array}{ll}
Q^{(k)} & q_{n}
\end{array}\right], \quad E^{(k)}=\left(Q^{(k)}\right)^{T} A P^{(k)} .
$$

6) Determine $l^{\star}$ from (3.39).

7) Decide whether to construct $v_{n+1}$ and $w_{n+1}$ as regular or inner vectors and go to 8 ) or 9), respectively.

8) Compute $\tilde{v}_{n+1}$ and $\tilde{w}_{n+1}$ by means of (3.42)-(3.43) and (3.40)-(3.41).

Set $n_{l+1}=n+1, l=l+1, V^{(l)}=W^{(l)}=0$ and go to 10$)$.

9) Compute $\tilde{v}_{n+1}$ and $\tilde{w}_{n+1}$ by means of (3.42), (3.44), and (3.40)-(3.41).

10) Compute $\rho_{n+1}=\left\|\tilde{v}_{n+1}\right\|$ and $\xi_{n+1}=\left\|\tilde{w}_{n+1}\right\|$.

If $\rho_{n+1}=0$ or $\xi_{n+1}=0$, then stop.

Otherwise, set

$$
\begin{aligned}
v_{n+1} & =\tilde{v}_{n+1} / \rho_{n+1}, \quad w_{n+1}=\tilde{w}_{n+1} / \xi_{n+1}, \\
V^{(l)} & =\left[\begin{array}{ll}
V^{(l)} & v_{n+1}
\end{array}\right], \quad W^{(l)}=\left[\begin{array}{ll}
W^{(l)} & w_{n+1}
\end{array}\right], \quad D^{(l)}=\left(W^{(l)}\right)^{T} V^{(l)} .
\end{aligned}
$$

We remark that Algorithm 3.3 reduces to the coupled two-term procedure described in $\S 3.2$ if all vectors in the $P-Q$ and $V-W$ sequences are built as regular vectors. Note that in this case, we have $k(n)=n-1, n_{k^{*}}=n-1, l(n)=n$, and $n_{l^{\star}}=n$ for all $n$. 
4. The look-ahead strategy. As described in $\S 3.2$, there are two possible breakdowns in the coupled two-term procedure without look-ahead: one associated with the $\mathrm{V}-\mathrm{W}$ sequence, and another associated with the $\mathrm{P}-\mathrm{Q}$ sequence. In particular, Algorithm 3.2 will encounter an exact breakdown in the $\mathrm{V}-\mathrm{W}$ sequence if $w_{n}^{T} v_{n}=0$, or in the P-Q sequence if $q_{n-1}^{T} A p_{n-1}=0$. The exact breakdowns of the two sequences are not independent of each other, as was pointed out by Gutknecht in [11]. For a full description of the structure and coupling of the exact breakdowns, we refer the reader to $[11,2]$ and the references given there. However, in practice one is also concerned with avoiding near-breakdowns, that is, situations when $w_{n}^{T} v_{n}$ or $q_{n-1}^{T} A p_{n-1}$ are not exactly zero, but are small in some sense.

In the coupled procedure with look-ahead, which we sketched in $\S 3.3$, exact and near-breakdowns in the $P-Q$ respectively $V-W$ sequence are prevented by building the next pair of vectors $p_{n}$ and $q_{n}$ respectively $v_{n+1}$ and $w_{n+1}$ as inner vectors. In this section, we describe the look-ahead strategy that is used to decide in steps 2) and 7) of Algorithm 3.3 whether vectors are constructed as regular or inner vectors.

Recall from $\S 2.2$ that the vectors $\left\{v_{i}\right\}_{i=1}^{n+1}$ and $\left\{w_{i}\right\}_{i=1}^{n+1}$ in the look-ahead Lanczos algorithm satisfy a block three-term recurrence that can be written compactly as (2.14)(2.15). By eliminating $V_{n}$ and $W_{n}$ in (3.7) and (3.8), one obtains a similar recurrence relation for the vectors $\left\{p_{i}\right\}_{i=1}^{n}$ and $\left\{q_{i}\right\}_{i=1}^{n}$ of the $P-Q$ sequence:

$$
A P_{n-1}=P_{n} U_{n} L_{n-1} \quad \text { and } A^{T} Q_{n-1}=Q_{n} \Gamma_{n}^{-1} U_{n} L_{n-1} \Gamma_{n-1} \text {. }
$$

By using the $A$-biorthogonality of $p_{n}$ and $q_{n}$, it is easy to show that the recurrences for the $\mathrm{P}-\mathrm{Q}$ sequence are also three-term recurrences, or, in the general case, block threeterm recurrences. We note that, in (4.1), the matrix $U_{n} L_{n-1}$ of recurrence coefficients is obtained by multiplying the factors $L_{n-1}$ and $U_{n}$ from the decompositions (3.11) of $H_{n-1}$ and $H_{n}$, respectively, in reverse order. This was first remarked by Rutishauser [16] for the special case of no look-ahead, and recently by Gutknecht [11], for the general case.

The look-ahead strategy consists of monitoring breakdowns in the two sequences independently. For the V-W sequence, the criteria used are the same as those proposed in [7]:

$$
\begin{gathered}
\sigma_{\min }\left(D^{(l)}\right) \geq \text { eps, } \\
n(A) \geq \sum_{i}\left|\left(L_{n} U_{n}\right)_{i n}\right|, \\
n(A) \geq \sum_{i} \frac{\gamma_{n}}{\gamma_{i}}\left|\left(L_{n} U_{n}\right)_{i n}\right|,
\end{gathered}
$$

where eps is machine epsilon, and $n(A)$ is an estimate for the norm of $A$. The Lanczos vectors $v_{n+1}$ and $w_{n+1}$ are built as regular vectors only if all three of the above checks are satisfied. The check (4.2) ensures that the diagonal blocks $D^{(j)}$ are nonsingular, while the checks (4.3)-(4.4) ensure that the size of the coefficients $\mu_{n}$ and $\nu_{n}$ in (2.23) 
and in the corresponding relation for $\tilde{w}_{n+1}$ do not exceed an estimate $n(A)$ for the norm of $A$. The first condition is needed since the inverse of $D^{(l)}$ appears in $\mu_{n}$, while the second and third conditions attempt to ensure that the components from $K_{n}\left(r_{0}, A\right)$ and from $K_{n}\left(w_{1}, A^{T}\right)$ do not dominate the $A v_{n}$ and $A^{T} w_{n}$ terms, respectively. Another motivation for these checks is as follows. The symmetric Lanczos process for Hermitian matrices $A$ generates tridiagonal matrices $H_{n}$ that satisfy

$$
\left\|H_{n}\right\| \leq\|A\| \text { for all } n \text {. }
$$

For the classical nonsymmetric Lanczos algorithm, the relation (4.5) does not hold in general. Indeed, formally, we have $\left\|H_{n}\right\|=\infty$ if a breakdown occurs. As David Day pointed out to us ${ }^{1}$, an "ideal" look-ahead Lanczos procedure would ensure that (4.5) also holds for non-Hermitian matrices. The criteria (4.3)-(4.4) can be viewed as a cheap way of modeling the conditions (4.5). We remark that the checks (4.3)-(4.4) take advantage of the normalization (2.9) of the Lanczos vectors.

For the $\mathrm{P}-\mathrm{Q}$ sequence, the criteria are similar: the diagonal blocks $E^{(j)}$ must be nonsingular, and the size of the last columns of $U_{n} L_{n-1}$ and of $\Gamma_{n}^{-1} U_{n} L_{n-1} \Gamma_{n-1}$ must not exceed the estimate $n(A)$ for the norm of $A$. Singularity of $E^{(k)}$ is once again checked from its smallest singular value:

$$
\sigma_{\min }\left(E^{(k)}\right) \geq \text { eps. }
$$

However, for the second and third checks, it is no longer sufficient to compute just the norm of the last column of the matrices of recurrence coefficients, as the vectors $p_{n}$ and $q_{n}$ are not normalized to unit length. Instead, one must check

$$
n(A)\left\|p_{n}\right\| \geq \sum_{i}\left|\left(U_{n} L_{n-1}\right)_{i, n-1}\right|\left\|p_{i}\right\|
$$

and

$$
n(A)\left\|q_{n}\right\| \geq \sum_{i} \frac{\gamma_{n-1}}{\gamma_{i}}\left|\left(U_{n} L_{n-1}\right)_{i, n-1}\right|\left\|q_{i}\right\| .
$$

This means that the look-ahead strategy for the $P-Q$ sequence requires the computation of the two norms $\left\|p_{n}\right\|$ and $\left\|q_{n}\right\|$ at each step, work that would otherwise not be needed by the algorithm. Once again, the vectors $p_{n}$ and $q_{n}$ are built as regular vectors only if all three of the above checks are satisfied. We remark that the look-ahead strategy presented here builds regular vectors in preference to inner vectors, and will therefore build as few inner vectors as possible.

Finally, we note that other look-ahead strategies are also possible. For example, Gutknecht [11] proposed a look-ahead strategy that assumes that the near-breakdowns encountered in the two sequences have the same structure as the exact breakdowns. We have chosen to monitor the two sequences independently; nevertheless, our strategy will recover the exact-breakdown structure if only exact breakdowns are considered.

${ }^{1}$ Private communication, Berkeley, March 1992. 
5. Implementation details. In this section, we present a detailed description of our implementation of the coupled two-term recurrence Lanczos algorithm with lookahead. We are interested in obtaining an implementation that requires only two inner products per iteration to compute all the coefficients of the recurrence formulas. Recall that the look-ahead strategy (4.6)-(4.7) for the P-Q sequence and the normalization (2.9) require a total of four norm computations, so that the implementation will require two inner products and four norms per iteration.

Let us denote by

$$
G_{n-1}:=U_{n} L_{n-1}
$$

the matrix of recurrence coefficients for the three-term recurrences for $P_{n}$ and $Q_{n}$ in (4.1). Let us also introduce the auxiliary matrices

$$
F_{n}:=W_{n}^{T} A P_{n} \quad \text { and } \quad \tilde{F}_{n}:=Q_{n}^{T} A V_{n},
$$

whose columns are needed in (3.35)-(3.36) and (3.42)-(3.43). It turns out that these two matrices are essentially the transpose of each other.

LEMMA 5.1. The matrices $F_{n}$ and $\tilde{F}_{n}$ satisfy:

$$
F_{n} \Gamma_{n}=\left(\tilde{F}_{n} \Gamma_{n}\right)^{T}
$$

Proof. The proof is similar to the proof of Lemma 3.1.

The $n$th iteration of the implementation will update the matrices $D_{n-1}, E_{n-1}, F_{n-1}$, $L_{n-1}, U_{n-1}, P_{n-1}, Q_{n-1}, V_{n}$, and $W_{n}$, to $D_{n}, E_{n}, F_{n}, L_{n}, U_{n}, P_{n}, Q_{n}, V_{n+1}$, and $W_{n+1}$, respectively.

We first list an outline of the algorithm as we have implemented it. This is essentially the same as Algorithm 3.3, up to the order of the steps and more details.

ALGORITHM 5.2. (Coupled algorithm with look-ahead)

0) Choose $v_{1}, w_{1} \in \mathrm{C}^{N}$ with $\left\|v_{1}\right\|=\left\|w_{1}\right\|=1$, and compute $w_{1}^{T} v_{1}$.

Set $k=1, m_{k}=1, l=1, n_{l}=1$.

For $n=1,2, \cdots$, do:

1) Update $D_{n-1}$ to $D_{n}$.

2) Determine $k^{\star}$ from (3.31):

$$
k^{\star}=\max \left\{j \mid 1 \leq j \leq k \text { and } m_{j} \leq \max \left\{1, n_{l}-1\right\}\right\} .
$$

3) Compute $F_{n, 1: n-1}$ from (5.2) below, using $L_{n-1}$ and $D_{n, 1: n}$.

Then compute $\tilde{F}_{1: n-1, n}$ by Lemma 5.1 .

4) Check whether $E^{(k)}$ is nonsingular:

$$
\text { innerp }=\sigma_{\min }\left(E^{(k)}\right)<\text { eps. }
$$


5) Compute the part of $U_{1: n, n}$ that is determined by biorthogonality:

$U_{m_{i}: m_{i+1}-1, n}=\left(E^{(i)}\right)^{-1}\left(Q^{(i)}\right)^{T} A v_{n}=\left(E^{(i)}\right)^{-1} \tilde{F}_{m_{i}: m_{i+1}-1, n}, \quad i=k^{\star}, \cdots, k-1$.

If innerp, go to 6). Otherwise, set

$$
U_{m_{k}: n-1, n}=\left(E^{(k)}\right)^{-1}\left(Q^{(k)}\right)^{T} A v_{n}=\left(E^{(k)}\right)^{-1} \tilde{F}_{m_{k}: n-1, n}
$$

6) Build the part of $p_{n}$ and $q_{n}$ that is common to both regular and inner vectors:

$$
\begin{aligned}
& \hat{p}_{n}=v_{n}-\sum_{i=m_{k^{\star}}}^{m_{k}-1} p_{i} u_{i n} \\
& \hat{q}_{n}=w_{n}-\sum_{i=m_{k^{*}}}^{m_{k}-1} q_{i} u_{i n}\left(\gamma_{n} / \gamma_{i}\right) .
\end{aligned}
$$

If innerp, go to 11).

7) Build and check the coefficient $G_{m_{k}: n-1, n-1}$. If innerp, go to 11).

8) Build $p_{n}$ and $q_{n}$ as regular vectors:

$$
\begin{aligned}
& p_{n}=\hat{p}_{n}-\sum_{i=m_{k}}^{n-1} p_{i} u_{i n} \\
& q_{n}=\hat{q}_{n}-\sum_{i=m_{k}}^{n-1} q_{i} u_{i n}\left(\gamma_{n} / \gamma_{i}\right)
\end{aligned}
$$

Compute $A p_{n}, q_{n}^{T} A p_{n},\left\|p_{n}\right\|$, and $\left\|q_{n}\right\|$.

9) Build and check the coefficient $G_{m_{k}: n-1, n}$. If innerp, go to 11).

10) Set $m_{k+1}=n, k=k+1$, and go to 12).

11) Choose the inner recurrence coefficients $u_{i n}, i=m_{k}, \cdots, n-1$, and build $p_{n}$ and $q_{n}$ as inner vectors:

$$
\begin{aligned}
& p_{n}=\hat{p}_{n}-\sum_{i=m_{k}}^{n-1} p_{i} u_{i n} \\
& q_{n}=\hat{q}_{n}-\sum_{i=m_{k}}^{n-1} q_{i} u_{i n}\left(\gamma_{n} / \gamma_{i}\right)
\end{aligned}
$$

Compute $A p_{n}, q_{n}^{T} A p_{n},\left\|p_{n}\right\|$, and $\left\|q_{n}\right\|$.

12) If $\left\|p_{n}\right\|=0$, or $\left\|q_{n}\right\|=0$, then stop.

13) Compute $A^{T} q_{n}$.

14) Update $E_{n-1}$ to $E_{n}$. 
15) Determine l from (3.39):

$$
l^{\star}=\max \left\{j \mid 1 \leq j \leq l \text { and } n_{j} \leq m_{k}\right\} .
$$

16) Compute $F_{1: n, n}$ from (5.3) below, using $E_{n}$ and $U_{n}$.

17) Check whether $D^{(l)}$ is nonsingular:

$$
\text { innerv }=\sigma_{\min }\left(D^{(l)}\right)<\text { eps. }
$$

18) Compute the part of $L_{1: n, n}$ that is determined by biorthogonality:

$$
L_{n_{i}: n_{i+1}-1, n}=\left(D^{(i)}\right)^{-1}\left(W^{(i)}\right)^{T} A p_{n}=\left(D^{(i)}\right)^{-1} F_{n_{i}: n_{i+1}-1, n}, \quad i=l^{\star}, \cdots, l-1,
$$

If innerv, go to 19). Otherwise, set

$$
L_{n_{1}: n, n}=\left(D^{(l)}\right)^{-1}\left(W^{(l)}\right)^{T} A p_{n}=\left(D^{(l)}\right)^{-1} F_{n_{l}: n, n} .
$$

19) Build the part of $v_{n+1}$ and $w_{n+1}$ that is common to both regular and inner vectors:

$$
\begin{aligned}
& \hat{v}_{n+1}=A p_{n}-\sum_{i=n_{l^{*}}}^{n_{l}-1} v_{i} l_{i n}, \\
& \hat{w}_{n+1}=A^{T} q_{n}-\sum_{i=n_{l^{*}}}^{n_{l}-1} w_{i} l_{i n}\left(\gamma_{n} / \gamma_{i}\right) .
\end{aligned}
$$

If innerv, go to 24).

20) Build and check the coefficient $H_{n_{i}: n, n}$. If innerv, go to 24).

21) Build $v_{n+1}$ and $w_{n+1}$ as regular vectors:

$$
\begin{aligned}
& \tilde{v}_{n+1}=\hat{v}_{n+1}-\sum_{i=n_{l}}^{n} v_{i} l_{i n}, \\
& \tilde{w}_{n+1}=\hat{w}_{n+1}-\sum_{i=n_{l}}^{n} w_{i} l_{i n}\left(\gamma_{n} / \gamma_{i}\right) .
\end{aligned}
$$

Compute $\rho_{n+1}=l_{n+1, n}=\left\|\tilde{v}_{n+1}\right\|, \xi_{n+1}=\left\|\tilde{w}_{n+1}\right\|$.

If $\rho_{n+1}=0$ or $\xi_{n+1}=0$, then stop.

Otherwise, set $\gamma_{n+1}=\gamma_{n} \rho_{n+1} / \xi_{n+1}$, and compute $\tilde{w}_{n+1}^{T} \tilde{v}_{n+1}$.

22) Build and check the coefficient $H_{n !: n, n+1}$. If innerv, go to 24).

23) Set $n_{l+1}=n+1, l=l+1$, and go to 25).

24) Choose the inner recurrence coefficients $l_{\text {in }}, i=n_{l}, \cdots, n$, and 
build $v_{n+1}$ and $w_{n+1}$ as inner vectors:

$$
\begin{aligned}
& \tilde{v}_{n+1}=\hat{v}_{n+1}-\sum_{i=n_{l}}^{n} v_{i} l_{i n} \\
& \tilde{w}_{n+1}=\hat{w}_{n+1}-\sum_{i=n_{l}}^{n} w_{i} l_{i n}\left(\gamma_{n} / \gamma_{i}\right) .
\end{aligned}
$$

Compute $\rho_{n+1}=l_{n+1, n}=\left\|\tilde{v}_{n+1}\right\|, \xi_{n+1}=\left\|\tilde{w}_{n+1}\right\|$.

If $\rho_{n+1}=0$ or $\xi_{n+1}=0$, then stop.

Otherwise, set $\gamma_{n+1}=\gamma_{n} \rho_{n+1} / \xi_{n+1}$, and compute $\tilde{w}_{n+1}^{T} \tilde{v}_{n+1}$.

25) Set

$$
\begin{gathered}
v_{n+1}=\tilde{v}_{n+1} / \rho_{n+1}, \quad w_{n+1}=\tilde{w}_{n+1} / \xi_{n+1} \\
w_{n+1}^{T} v_{n+1}=\tilde{w}_{n+1}^{T} \tilde{v}_{n+1} /\left(\rho_{n+1} \xi_{n+1}\right)
\end{gathered}
$$

Step 1. The diagonal term $w_{n}^{T} v_{n}$ has already been computed directly, at the end of the previous step. Next, using

$$
\begin{aligned}
F_{n-1} & =W_{n-1}^{T} A P_{n-1}=W_{n-1}^{T} V_{n} L_{n-1} \\
& =D_{n-1} L_{1: n-1,1: n-1}+l_{n, n-1} D_{1: n-1, n}\left[\begin{array}{llll}
0 & \cdots & 0 & 1
\end{array}\right],
\end{aligned}
$$

the remainder of the last column of $D_{n}$ is computed from $D_{n-1}, F_{n-1}$, and $L_{n-1}$. The last row of $D_{n}$ is obtained by symmetry, using (3.15) from Lemma 3.1.

Step 7. We build $G_{m_{k}: n-1, n-1}$, which would be the coefficient of the $P^{(k)}$ and $Q^{(k)}$ blocks in the three-term recurrences for $p_{n}$ and $q_{n}$. Using (5.1), one has:

$$
G_{i, n-1}=\sum_{j=i}^{n} u_{i j} l_{j, n-1}, \quad i=m_{k}, \cdots, n-1 .
$$

We then check (4.6)-(4.7), and set innerp to TRUE if at least one of the two checks fails.

Step 9. We build $G_{m_{k}: n-1, n}$, which would be the coefficient of the $P^{(k)}$ and $Q^{(k)}$ blocks in the three-term recurrences for $p_{n+1}$ and $q_{n+1}$. It is straightforward to show that

$$
G_{m_{k}: n-1, n}=\left(E^{(k)}\right)^{-1}\left(Q^{(k)}\right)^{T} A A p_{n}
$$

Moreover, we have

$$
\begin{aligned}
Q_{n-1}^{T} A A p_{n} & =\left(A^{T} Q_{n-1}\right)^{T} A p_{n}=\left(Q_{n} \Gamma_{n}^{-1} U_{n} L_{n-1} \Gamma_{n-1}\right)^{T} A p_{n} \\
& =\Gamma_{n-1} L_{n-1}^{T} U_{n}^{T} \Gamma_{n}^{-1} Q_{n}^{T} A p_{n} \\
& =\Gamma_{n-1} L_{n-1}^{T} U_{n}^{T} \Gamma_{n}^{-1}\left[\begin{array}{llll}
0 & \cdots & 0 & q_{n}^{T} A p_{n}
\end{array}\right]^{T} \\
& =\frac{\gamma_{n-1}}{\gamma_{n}} l_{n, n-1}\left[\begin{array}{lllll}
0 & \cdots & 0 & q_{n}^{T} A p_{n}
\end{array}\right]^{T}
\end{aligned}
$$


We then check (4.6)-(4.7), and set innerp to TRUE if at least one of the two checks fails.

Step 14. The diagonal term $q_{n}^{T} A p_{n}$ has already been computed directly, as part of Step 8) or Step 11). Next, using

$$
F_{n}=W_{n}^{T} A P_{n}=\Gamma_{n} U_{n}^{T} \Gamma_{n}^{-1} Q_{n}^{T} A P_{n},
$$

the remainder of the last row of $E_{n}$ is computed from $E_{n-1}, F_{1: n, 1: n-1}$, and $U_{n}$. The last column of $E_{n}$ is obtained by symmetry, using (3.16) from Lemma 3.1.

Step 20. We build $H_{n_{l}: n, n}$, which would be the coefficient of the $V^{(l)}$ and $W^{(l)}$ blocks in the three-term recurrences for $v_{n+1}$ and $w_{n+1}$. Using (3.11), one has:

$$
H_{i n}=\sum_{j=i}^{n} l_{i j} u_{j n}, \quad i=n_{l}, \cdots, n
$$

We then check (4.3)-(4.4), and set innerv to TRUE if at least one of the two checks fails.

Step 22. We build $H_{n_{1}: n, n+1}$, which would be the coefficient of the $V^{(l)}$ and $W^{(l)}$ blocks in the three-term recurrences for $v_{n+2}$ and $w_{n+2}$. It is straightforward to show that

$$
H_{n ! n, n+1}=\left(D^{(l)}\right)^{-1}\left(W^{(l)}\right)^{T} A v_{n+1}
$$

Moreover, we have

$$
\begin{aligned}
W_{n}^{T} A v_{n+1} & =\left(A^{T} W_{n}\right)^{T} A v_{n+1}=\left(W_{n+1} \Gamma_{n+1}^{-1} L_{n} U_{n} \Gamma_{n}\right)^{T} v_{n+1} \\
& =\Gamma_{n} U_{n}^{T} L_{n}^{T} \Gamma_{n+1}^{-1} W_{n+1}^{T} v_{n+1} \\
& =\Gamma_{n} U_{n}^{T} L_{n}^{T} \Gamma_{n+1}^{-1}\left[\begin{array}{llll}
0 & \cdots & 0 & w_{n+1}^{T} v_{n+1}
\end{array}\right]^{T} \\
& =\frac{\gamma_{n}}{\gamma_{n+1}} l_{n+1, n}\left[\begin{array}{llll}
0 & \cdots & 0 & w_{n+1}^{T} v_{n+1}
\end{array}\right]^{T} .
\end{aligned}
$$

We then check (4.3)-(4.4), and set innerv to TRUE if at least one of the two checks fails.

We remark that the checks in steps 7) and 9), and 20) and 22), are actually slightly relaxed versions of (4.3)-(4.4), and (4.6)-(4.7), respectively, since the indices checked are only a subset of the full range appearing in (4.3)-(4.4) and (4.6)-(4.7). We also note that the algorithm above requires minimal inputs from the user. Recall that eps in steps 4) and 17) is machine epsilon. Furthermore, the estimate $n(A)$ for the norm of the matrix can be updated dynamically, as was done in [7].

The coupled Lanczos Algorithm 5.2 requires per iteration the computation of two inner products and four vector norms. We conclude this section by noting that, in $\mathrm{Al}-$ gorithm 5.2, the choice of the inner recurrence coefficients (3.37) and (3.44) is arbitrary. 
In our implementation of the algorithm, we have used

$$
\begin{aligned}
u_{n-1, n} & =1, \\
u_{n-2, n} & =1, \quad \text { when } \quad m_{k} \leq n-2, \\
u_{i n} & =0, \quad \text { for } i=m_{k}, \cdots, n-3, \\
l_{n n} & =1, \\
l_{n-1, n} & =1, \quad \text { when } \quad n_{l} \leq n-1, \\
l_{\text {in }} & =0, \quad \text { for } i=n_{l}, \cdots, n-2,
\end{aligned}
$$

for the inner vector recurrence coefficients.

6. An implementation of QMR with look-ahead. We now return to linear systems (1.1) and the QMR method. In this section, we propose an implementation of QMR method based on the coupled two-term look-ahead Algorithm 3.3.

Recall that the $n$th QMR iterate $x_{n}$ is defined by (2.25)-(2.26) in terms of the matrices $V_{n}$ and $H_{n}$ generated by the look-ahead Lanczos algorithm. In the original implementation of QMR, the solution $z_{n}$ of the least squares problem (2.26) is computed by means of a QR decomposition of the matrix $\Omega_{n+1} H_{n}$.

Here we consider the case that the Lanczos vectors are constructed using the coupled two-term Algorithm 3.3. Recall that Algorithm 3.3 yields as a by-product the factors $L_{n}$ and $U_{n}$ in the decomposition (3.11) of $H_{n}$. Using the factorization (3.11) and setting $y_{n}:=U_{n} z_{n}$, we can rewrite the definition $(2.25)-(2.26)$ of $x_{n}$ as follows:

$$
x_{n}=x_{0}+V_{n} U_{n}^{-1} y_{n},
$$

where $y_{n}$ is the unique solution of the least squares problem

$$
\left\|f_{n+1}-\Omega_{n+1} L_{n} y_{n}\right\|=\min _{y \in \mathbb{C}^{n}}\left\|f_{n+1}-\Omega_{n+1} L_{n} y\right\| .
$$

Here, as before, $f_{n+1}$ is given by (2.27) and $\Omega_{n+1}$ is defined in (2.28). We remark that the least squares problem (6.2) is actually cheaper to solve than the original one (2.26). The reason is that the matrix $L_{n}$ in (6.2) has fewer nonzero elements than $H_{n}$ in (2.26). For example, if no look-ahead steps are taken, then $H_{n}$ is tridiagonal, while $L_{n}$ is a lower bidiagonal matrix. This special case will be considered in more detail in $\S 7$.

As discussed in [9], solutions of least squares problems of the type (6.2) can be easily updated from step to step, using the QR decomposition of $\Omega_{n+1} L_{n}$,

$$
\Omega_{n+1} L_{n}=Q_{n}^{H}\left[\begin{array}{c}
R_{n} \\
0
\end{array}\right]
$$

where $Q_{n}$ is a unitary $(n+1) \times(n+1)$ matrix, and $R_{n}$ is a nonsingular upper triangular $n \times n$ matrix. With this, the least squares problem (6.2) becomes

$$
\begin{aligned}
\min _{y \in \mathrm{C}^{n}}\left\|f_{n+1}-\Omega_{n+1} L_{n} y\right\| & =\min _{y \in \mathrm{C}^{n}}\left\|Q_{n}^{H}\left(Q_{n} f_{n+1}-\left[\begin{array}{c}
R_{n} \\
0
\end{array}\right] y\right)\right\| \\
& =\min _{y \in \mathrm{C}^{n}}\left\|Q_{n} f_{n+1}-\left[\begin{array}{c}
R_{n} \\
0
\end{array}\right] y\right\| .
\end{aligned}
$$


This gives for $y_{n}$ :

$$
y_{n}=\left(R_{n}\right)^{-1} t_{n}, \quad \text { where } \quad t_{n}=\left[\begin{array}{c}
\tau_{1} \\
\vdots \\
\tau_{n}
\end{array}\right], \quad\left[\begin{array}{c}
t_{n} \\
\tilde{\tau}_{n+1}
\end{array}\right]:=Q_{n} f_{n+1} .
$$

Finally, we note that it is possible to update the QMR iterate at each step, as was done in the original QMR algorithm. Full implementation details were given in [9, Sect. 4], and we will not repeat them here. The point is that the QMR iterates have an update formula of the form

$$
x_{n}=x_{n-1}+d_{n} \tau_{n} .
$$

Here, $\tau_{n}$ is given by (6.4), and $d_{n}$ is an auxiliary search direction defined as the last column of the matrix $V_{n} U_{n}^{-1} R_{n}^{-1}$. The vectors $d_{n}$ are also updated with short recurrences: the recurrence for $d_{n}$ involves only as many vectors as the recurrence for $v_{n+1}$. For full details of the update procedure for $d_{n}$, we refer the reader to [9].

The basic outline of the resulting implementation of QMR based on the coupled two-term Algorithm 3.3 is then as follows.

AlgoRITHM 6.1. (QMR based on coupled recurrences)

0) Choose $x_{0} \in C^{N}$ and set $r_{0}=b-A x_{0}, \rho_{1}=\left\|r_{0}\right\|, v_{1}=r_{0} / \rho_{1}$.

Choose $w_{1} \in C^{N}$ with $\left\|w_{1}\right\|=1$.

For $n=1,2, \cdots$, do:

1) Perform the nth iteration of the coupled two-term Algorithm 3.3.

This yields matrices $L_{n}, P_{n}, U_{n}$, and $V_{n+1}$, which satisfy (3.7).

2) Update the $Q R$ factorization (6.3) of $\Omega_{n+1} L_{n}$ and the vector $t_{n}$ in (6.4).

3) Update the QMR iterate $x_{n}$ by means of (6.5).

4) If $x_{n}$ has converged, then stop.

In [9], various properties of the QMR method are given. For example, it is shown how existing BCG iterates can be easily recovered from the QMR process, and how estimates for the QMR residual norms can be obtained at no extra costs. We would like to stress that these properties also hold true for the particular implementation of QMR sketched in Algorithm 6.1.

7. An implementation of QMR without look-ahead. In this section, we present the simplification of Algorithm 6.1 to the case where no look-ahead is used. We also briefly address the issue of preconditioning.

Let $M$ be a given nonsingular $N \times N$ matrix that approximates in some sense the coefficient matrix $A$ of (1.1). Suppose further that $M$ is decomposed as

$$
M=M_{1} M_{2} \text {. }
$$

Then, one applies the QMR algorithm to the system

$$
A^{\prime} y^{\prime}=b^{\prime}
$$


where $A^{\prime}=M_{1}^{-1} A M_{2}^{-1}, b^{\prime}=M_{1}^{-1} b$, and $x^{\prime}=M_{2} x$. It is easy to see that the linear systems (1.1) and (7.2) are equivalent, and that one can transform back from the iterates $x_{n}^{\prime}$ and the residuals $r_{n}^{\prime}$ of the system (7.1) to the iterates $x_{n}$ and the residuals $r_{n}$ of the system (1.1). For example, while applying QMR to the preconditioned system (7.2), it is possible to write the resulting algorithm in terms of the quantities corresponding to the original system (1.1); this is what is done below.

We now present a version of the QMR algorithm based on the coupled Algorithm 3.2, which does not have look-ahead. We remark that in this case, by (3.22) and (3.20), the matrix $U_{n}$ in (3.9) is upper bidiagonal, and $L_{n}$ is a lower bidiagonal matrix. We also implement preconditioning, as discussed above. The resulting QMR algorithm is as follows.

ALGORITHM 7.1. (QMR based on coupled recurrences without look-ahead)

0) Choose $x_{0} \in \mathrm{C}^{N}$ and set $r_{0}=b-A x_{0}$.

Compute $\rho_{1}=\left\|M_{1}^{-1} r_{0}\right\|$ and set $v_{1}=r_{0} / \rho_{1}$.

Choose $w_{1} \in \mathrm{C}^{N}$ with $\left\|M_{2}^{-T} w_{1}\right\|=1$.

Set $p_{0}=q_{0}=d_{0}, c_{0}=\epsilon_{0}=\xi_{1}=1, \vartheta_{0}=0, \eta_{0}=-1$.

For $n=1,2, \cdots$, do:

1) If $\epsilon_{n-1}=0$, then stop.

Compute $\delta_{n}=w_{n}^{T} M^{-1} v_{n}$.

If $\delta_{n}=0$, then stop.

2) Compute

$$
\begin{aligned}
& p_{n}=M^{-1} v_{n}-p_{n-1}\left(\xi_{n} \delta_{n} / \epsilon_{n-1}\right), \\
& q_{n}=M^{-T} w_{n}-q_{n-1}\left(\rho_{n} \delta_{n} / \epsilon_{n-1}\right) .
\end{aligned}
$$

3) Compute $\epsilon_{n}=q_{n}^{T} A p_{n}, \beta_{n}=\epsilon_{n} / \delta_{n}$, and set

$$
\begin{gathered}
\tilde{v}_{n+1}=A p_{n}-v_{n} \beta_{n}, \quad \rho_{n+1}=\left\|M_{1}^{-1} \tilde{v}_{n+1}\right\|, \\
\tilde{w}_{n+1}=A^{T} q_{n}-w_{n} \beta_{n}, \quad \xi_{n+1}=\left\|M_{2}^{-T} \tilde{w}_{n+1}\right\| .
\end{gathered}
$$

4) Compute

$$
\begin{aligned}
& \vartheta_{n}=\frac{\omega_{n+1} \rho_{n+1}}{\omega_{n} c_{n-1}\left|\beta_{n}\right|}, \quad c_{n}=\frac{1}{\sqrt{1+\vartheta_{n}^{2}}}, \quad \eta_{n}=-\eta_{n-1} \frac{\rho_{n} c_{n}^{2}}{\beta_{n} c_{n-1}^{2}}, \\
& d_{n}=p_{n} \eta_{n}+d_{n-1}\left(\vartheta_{n-1} c_{n}\right)^{2}, \quad x_{n}=x_{n-1}+d_{n} .
\end{aligned}
$$

5) If $\rho_{n+1}=0$ or $\xi_{n+1}=0$, then stop.

Otherwise, set

$$
v_{n+1}=\tilde{v}_{n+1} / \rho_{n+1}, \quad w_{n+1}=\tilde{w}_{n+1} / \xi_{n+1}
$$


As pointed out in $\S 3.2$, the vectors computed in steps 2) and 3) of Algorithm 7.1 are rescaled versions of vectors used in BCG. Freund and Szeto [10] have used this connection to derive an implementation of QMR without look-ahead that is directly based on BCG. Their Algorithm 3.1 in [10] and Algorithm 7.1 are mathematically equivalent.

8. QMR for complex symmetric matrices. In this section, we briefly discuss the application of the QMR Algorithm 7.1 to the solution of complex symmetric linear systems, i.e., systems with

$$
A=A^{T} \in \mathrm{C}^{N \times N} \text {. }
$$

We note that the QMR approach was originally proposed by Freund in [5] for exactly this class of linear systems.

The benefit of applying a Lanczos method to complex symmetric systems is that the underlying Lanczos algorithm simplifies naturally. Recall that in the coupled Algorithm 3.3, the second starting vector $w_{1}$ is arbitrary. In the case of a symmetric matrix, if $w_{1}$ is chosen equal to $v_{1}$, then it is easy to show that $w_{n}=v_{n}$ and $q_{n}=p_{n}$, for all $n$. Thus, the recurrences for $w_{n}$ and $q_{n}$ can be eliminated, saving roughly half the amount of work and storage.

However, we remark that-in contrast to the Lanczos algorithm for Hermitian matrices, where breakdowns are excluded-the Lanczos process for complex symmetric also requires look-ahead in order to avoid exact and near-breakdowns. This is discussed in detail in [5].

The only other issue in the case of complex symmetric systems is that the preconditioner $M$ in (7.1) must also be symmetric, i.e.,

$$
M=M_{1} M_{2}=\left(M_{1} M_{2}\right)^{T}=M^{T} \text {. }
$$

For example, this is always guaranteed if the decomposition (7.1) is "symmetric" in the sense that

$$
M_{2}=M_{1}^{T}
$$

However, we stress that the condition (8.2) is not necessary, and $M_{1}$ and $M_{2}$ can be arbitrary matrices satisfying (8.1). We remark that standard preconditioning techniques, such as incomplete factorization, yield symmetric preconditioners (8.1) when applied to symmetric matrices $A$.

In this case, one can apply the QMR Algorithm 7.1 to the resulting preconditioned system. Once again writing everything in terms of the quantities corresponding to the original system (1.1), one obtains the following iteration. 
ALGORITHM 8.1. (QMR without look-ahead for complex symmetric systems)

0) Choose $x_{0} \in C^{N}$ and set $r_{0}=b-A x_{0}$.

Compute $\rho_{1}=\left\|M_{1}^{-1} r_{0}\right\|$ and set $v_{1}=r_{0} / \rho_{1}$.

Set $p_{0}=d_{0}, c_{0}=\epsilon_{0}=1, \vartheta_{0}=0, \eta_{0}=-1$.

For $n=1,2, \cdots, d o$ :

1) If $\epsilon_{n-1}=0$, then stop.

Compute $\delta_{n}=v_{n}^{T} M^{-1} v_{n}$.

If $\delta_{n}=0$, then stop.

2) Compute

$$
p_{n}=M^{-1} v_{n}-p_{n-1}\left(\rho_{n} \delta_{n} / \epsilon_{n-1}\right) .
$$

3) Compute $\epsilon_{n}=p_{n}^{T} A p_{n}, \beta_{n}=\epsilon_{n} / \delta_{n}$, and

$$
\tilde{v}_{n+1}=A p_{n}-v_{n} \beta_{n}, \quad \rho_{n+1}=\left\|M_{1}^{-1} \tilde{v}_{n+1}\right\| .
$$

4) Compute

$$
\begin{aligned}
& \vartheta_{n}=\frac{\omega_{n+1} \rho_{n+1}}{\omega_{n} c_{n-1}\left|\beta_{n}\right|}, \quad c_{n}=\frac{1}{\sqrt{1+\vartheta_{n}^{2}}}, \quad \eta_{n}=-\eta_{n-1} \frac{\rho_{n} c_{n}^{2}}{\beta_{n} c_{n-1}^{2}} \\
& d_{n}=p_{n} \eta_{n}+d_{n-1}\left(\vartheta_{n-1} c_{n}\right)^{2}, \quad x_{n}=x_{n-1}+d_{n} .
\end{aligned}
$$

5) If $\rho_{n+1}=0$, then stop.

Otherwise, set

$$
v_{n+1}=\tilde{v}_{n+1} / \rho_{n+1} .
$$

9. Numerical experiments. In this section, we present a few numerical examples. We compare the original and the new implementation of the QMR algorithm, as well as illustrate an application of the coupled QMR algorithm to the solution of complex symmetric linear systems.

In Figures 9.1-9.3 below, we always show the true relative residual norm $\left\|r_{n}\right\| /\left\|r_{0}\right\|$ plotted versus the iteration index $n$.

Example 9.1. This example is taken from [1], and is meant to illustrate the typical behavior that can be expected from the new implementation of QMR when compared to the original implementation. We consider the differential equation

$$
L u=f \text { on }(0,1) \times(0,1),
$$

where

$$
\begin{aligned}
L u:= & -\frac{\partial}{\partial x}\left(e^{-x y} \frac{\partial u}{\partial x}\right)-\frac{\partial}{\partial y}\left(e^{x y} \frac{\partial u}{\partial y}\right) \\
& +20(x+y) \frac{\partial u}{\partial x}+20 \frac{\partial}{\partial x}((x+y) u)+\frac{1}{1+x+y} u
\end{aligned}
$$




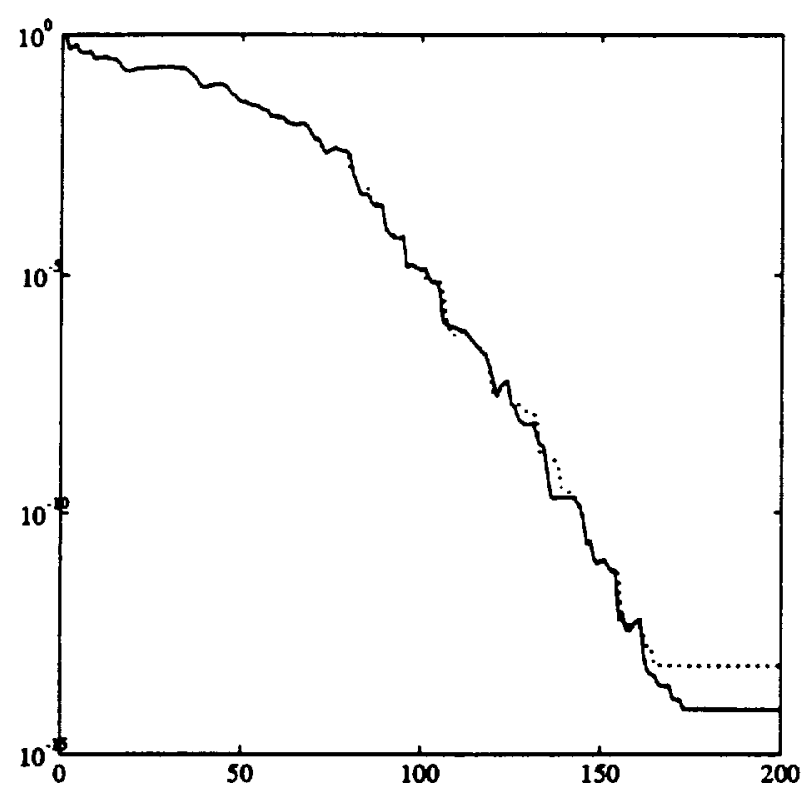

Fig. 9.1. Convergence curves for Example 9.1.

with Dirichlet boundary conditions $u=0$. We discretized (9.1) using centered differences on a $29 \times 29$ grid with mesh size $h=1 / 30$. This leads to a linear system $A x=b$, where $A$ is a nonsymmetric matrix of order $N=900$ with 4380 nonzero entries. We ran the original and the new implementations of QMR, both with look-ahead and with the same starting conditions, until the true residual norm $\left\|r_{n}\right\|$ was not reduced any further. The vectors $b$ and $w_{1}$ were random vectors, the initial guess $x_{0}$ was zero, and the example was run without preconditioning. The original QMR algorithm is plotted with a dotted line; it stagnated at $6.7 \mathrm{E}-14$, and it built 6 look-ahead blocks of size 2 . The coupled QMR algorithm is plotted with a solid line; it stagnated at $8.3 \mathrm{E}-15$, and it built 4 blocks of size 2 in the $V-W$ sequence and 7 blocks of size 2 in the $P-Q$ sequence. This behavior seems to be fairly typical, in that usually the new implementation is better than the original implementation, but the difference is not very large.

However, there are cases where the coupled implementation is significantly better than the original QMR implementation. The next example is of this type.

Example 9.2. This is a linear system that arises in performance modeling of multiprocessor systems, using Petri net analysis. In such applications, one obtains large sparse singular matrices $A$ with null spaces of dimension 1 , and one needs to compute a nontrivial basis vector for this null space. This leads to a linear system of the form

$$
A x=0,
$$

and thus condition (2.1) is satisfied. We used a matrix $A$ of size $N=3663$ with 23397 non-zero elements. The vector $b$ was zero, while the initial guess $x_{0}$ and the starting 


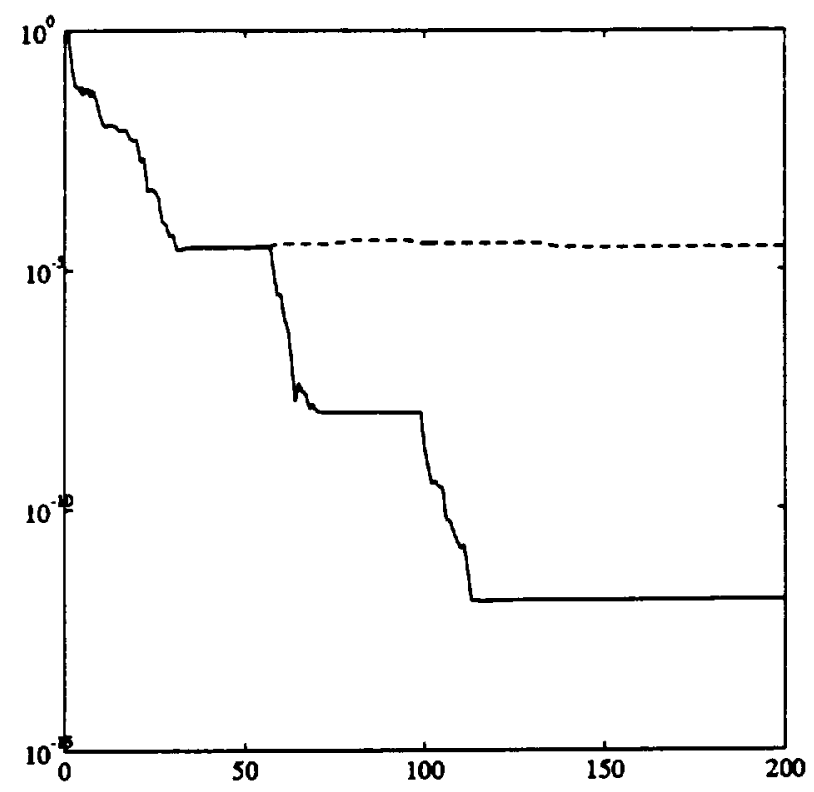

Fig. 9.2. Convergence curves for Example 9.2.

vector $w_{1}$ were both random. The linear system is a difficult one and does not converge easily without preconditioning. We used the variant described in [9] of Saad's ILUT preconditioner [17], with no additional fill-in allowed and a drop tolerance of 0.001 , which generated a preconditioning matrix $M$ with 23397 elements. The original QMR algorithm is plotted with a dotted line; it stagnated at $2.9 \mathrm{E}-5$, and it built 2 blocks of size 2 . On the other hand, the new implementation, plotted with a solid line, stagnated at $1.2 \mathrm{E}-12$, and it built 1 block of size 2 in the $\mathrm{V}-\mathrm{W}$ sequence and 3 blocks of size 2 in the $P-Q$ sequence.

Example 9.3. Here $A$ is the complex symmetric YOUNG1C matrix from the Harwell-Boeing test collection of sparse matrices [3]. The matrix arises in a scattering problem in aerodynamics research; it is of dimension $N=841$ with 4089 non-zero elements. We ran Algorithm 8.1, with various complex symmetric ILUT preconditioners. In all cases, the iteration was started with the same random vector for $b$ and zero initial guess $x_{0}$. This system is also a difficult one, and if not preconditioned, the QMR algorithm requires around 700 iterations to reach the stagnation level of $2.5 \mathrm{E}-14$; the corresponding convergence curve is plotted in a dashed line. However, the ILUT preconditioner is quite effective in this example, especially at higher levels of allowed fill-in and/or drop tolerance. The graph shows, in order of solid lines from right to left, ILUT with no additional fill-in and 0.001 drop tolerance (2375 non-zero elements), ILUT with 5 additional fill-in and 0.001 drop tolerance (5171 non-zero elements), ILUT with 10 additional fill-in and 0.001 drop tolerance (9320 non-zero elements), and finally ILUT with 16 additional fill-in and 0.0 drop tolerance (13329 non-zero elements). As can be 


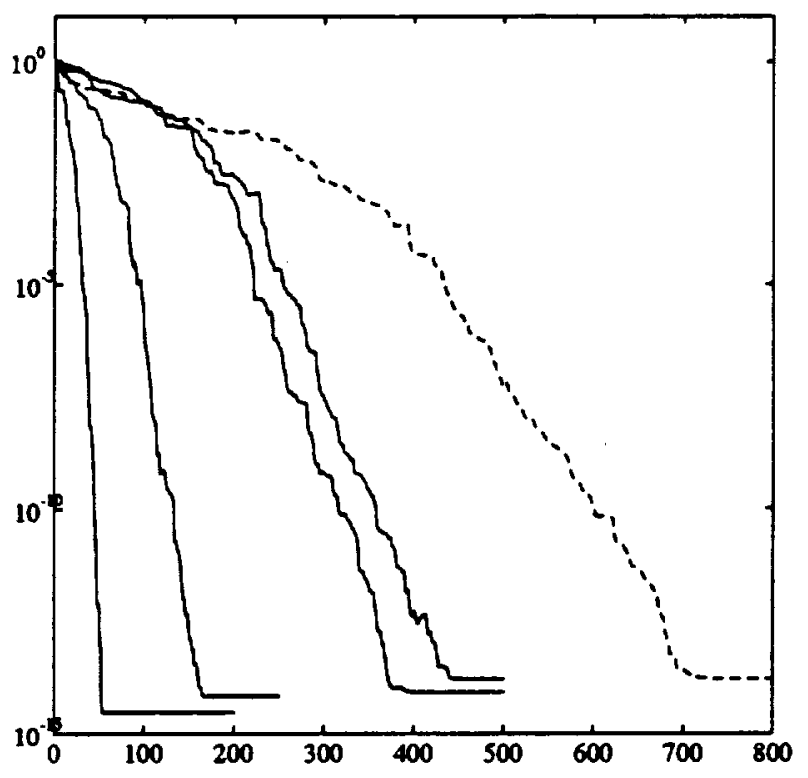

Fig. 9.3. Convergence curves for Example 9.3.

seen, all variants reach roughly the same stagnation level, around $1.0 \mathrm{E}-14$. However, they do so in fewer and fewer iterations, and in fact, for this example, the additional time spent computing the denser preconditioners was always made up by converging in fewer iterations.

10. Concluding remarks. We have presented a new look-ahead algorithm for constructing Lanczos vectors based on coupled two-term recurrences instead of the usual three-term recurrences. We then discussed a new implementation of the quasiminimal residual algorithm, using the coupled process to build the basis for the Krylov space. While the theoretical results derived for the original algorithm carry over to the new one, the latter was shown in examples to have better numerical properties. We also briefly covered an implementation of the new QMR method without look-ahead, as well as the application of the QMR algorithm to the solution of complex symmetric linear systems, where the underlying Lanczos process naturally simplifies.

FORTRAN 77 codes for the proposed coupled-two term look-ahead procedure and the resulting new implementation of the QMR algorithm can be obtained electronically from the authors (freund@research.att.com or na.nachtigal@na-net.ornl.gov). We note that FORTRAN 77 codes for the original implementation of QMR and the underlying look-ahead Lanczos algorithm are available from netlib by sending an email message consisting of the single line "send lalqmr from misc" to netlib@ornl.gov or netlib@research.att.com. 
Acknowledgement. The authors wish to acknowledge the fruitful discussions held with Martin Gutknecht and Tedd Szeto. Marlis Hochbruck and Uwe Seidel have provided the matrix for Example 9.2. Finally, we would like to thank Susanne Freund for her careful reading of the manuscript.

\section{REFERENCES}

[1] J. K. Cullum AND R. A. WilloughBY, A practical procedure for computing eigenvalues of large sparse nonsymmetric matrices, in Large Scale Eigenvalue Problems, J. K. Cullum and R. A. Willoughby, eds., North-Holland, 1986, pp. 193-240.

[2] A. Draux, Polynômes Orthogonaux Formels - Applications, Lecture Notes in Mathematics 974, Springer-Verlag, Berlin, Germany, 1983.

[3] I. S. Duff, R. G. Grimes, AND J. G. Lewis, Sparse matrix test problems, ACM Trans. Math. Software, 15 (1989), pp. 1-14.

[4] R. FLETCHER, Conjugate gradient methods for indefinite systems, in Numerical Analysis Dundee 1975 , G. A. Watson, ed., Lecture Notes in Mathematics 506, Berlin, Germany, 1976, SpringerVerlag, pp. 73-89.

[5] R. W. FREUND, Conjugate gradient-type methods for linear systems with complex symmetric coefficient matrices, SIAM J. Sci. Statist. Comput., 13 (1992), pp. 425-448.

[6] - Quasi-kernel polynomials and convergence results for quasi-minimal residual iterations, Technical Report 92.07, RIACS, NASA Ames Research Center, Moffett Field, CA, March 1992. To appear in Numerical Methods of Approximation Theory.

[7] R. W. Freund, M. H. GutKnecht, AND N. M. Nachtigal, An implementation of the lookahead Lanczos algorithm for non-Hermitian matrices, Technical Report 91.09, RIACS, NASA Ames Research Center, Moffett Field, CA, April 1991. To appear in the SIAM Journal on Scientific and Statistical Computing.

[8] R. W. FREUND AND M. HochBRUCK, On the use of two QMR algorithms for solving singular systems and applications in Markov chain modeling, Technical Report 91.25, RIACS, NASA Ames Research Center, Moffett Field, CA, December 1991.

[9] R. W. FREUND AND N. M. NACHTIGAL, QMR: a quasi-minimal residual method for nonHermitian linear systems, Numer. Math., 60 (1991), pp. 315-339.

[10] R. W. FreUnd AND T. SzETo, A quasi-minimal residual squared algorithm for non-Hermitian linear systems. Proceedings of the 1992 Copper Mountain Conference on Iterative Methods, April 1992.

[11] M. H. GuTKNecht, A completed theory of the unsymmetric Lanczos process and related algorithms, Part II, IPS Research Report 90-16, IPS, ETH, Zürich, Switzerland, September 1990.

$[12]-$, A completed theory of the unsymmetric Lanczos process and related algorithms, Part $I$, SIAM J. Matrix Anal. Appl., 13 (1992), pp. 594-639.

[13] C. Lanczos, An iteration method for the solution of the eigenvalue problem of linear differential and integral operators, J. Res. Nat. Bur. Standards, 45 (1950), pp. 255-282.

[14] - Solution of systems of linear equations by minimized iterations, J. Res. Nat. Bur. Standards, 49 (1952), pp. 33-53.

[15] B. N. PARLETT, D. R. TAYLOR, AND Z. A. LiU, A look-ahead Lanczos algorithm for unsymmetric matrices, Math. Comp., 44 (1985), pp. 105-124.

[16] H. RuTISHaUSER, Der Quotienten-Differenzen-Algorithmus, vol. 7 of Mitteilungen aus dem Institut für angewandte Mathematik an der ETH Zürich, Birkhaüser, Basel, Switzerland, 1957.

[17] Y. SAAD, ILUT: a dual threshold incomplete $L U$ factorization, Research Report UMSI 92/38, University of Minnesota Supercomputer Institute, Minneapolis, MN, March 1992.

[18] D. R. TAYLOR, Analysis of the look ahead Lanczos algorithm, PhD Thesis, Department of Mathematics, University of California, Berkeley, CA, November 1982. 
